\title{
Size distribution of circumstellar disks in the Trapezium cluster ${ }^{\star}$
}

\author{
S. M. Vicente ${ }^{1,2}$ and J. Alves ${ }^{1}$ \\ 1 European Southern Observatory, Karl-Schwarzschild Straße 2, 85748 Garching bei München, Germany \\ e-mail: [svicente; jalves]@eso.org \\ ${ }^{2}$ Faculdade de Ciências da Universidade de Lisboa, Campo Grande, Portugal
}

Received 31 May 2005 / Accepted 3 June 2005

\begin{abstract}
In this paper we present results on the size distribution of circumstellar disks in the Trapezium cluster as measured from HST/WFPC2 data. Direct diameter measurements of a sample of 135 bright proplyds and 14 silhouettes disks suggest that there is a single population of disks well characterized by a power-law distribution with an exponent of $-1.9 \pm 0.3$ between disk diameters 100-400 AU. For the stellar mass sampled (from late $\mathrm{G}$ to late $\mathrm{M}$ stars) we find no obvious correlation between disk diameter and stellar mass. We also find that there is no obvious correlation between disk diameter and the projected distance to the ionizing Trapezium OB stars. We estimate that about $40 \%$ of the disks in the Trapezium have radius larger than 50 AU. We suggest that the origin of the Solar system's (Kuiper belt) outer edge is likely to be due to the star formation environment and disk destruction processes (photoevaporation, collisions) present in the stellar cluster on which the Sun was probably formed. Finally, we identified a previously unknown proplyd and named it 266-557, following convention.
\end{abstract}

Key words. stars: planetary systems: protoplanetary disks - stars: planetary systems: formation - stars: pre-main sequence stars: low-mass, brown dwarfs - stars: formation

\section{Introduction}

Understanding the formation of planets from protoplanetary disks surrounding young stars is an important goal of modern day astrophysics. Although there is evidence for most stars in our Galaxy to have formed in dense photo-evaporating clusters much like the Trapezium cluster (e.g., Lada \& Lada 2003), it is not yet clear that the circumstellar disks once associated with these stars survived the harsh conditions imposed by massive star formation, and matured into planetary systems. The Trapezium cluster in the Orion star forming region (M42, NGC 1976) is a unique laboratory to tackle this problem, mainly because it is the closest $(d=450$ pc, e.g., Muench et al. 2002) massive star forming region. It is also very young ( 1 Myr) (Hillenbrand 1997; Hillenbrand \& Carpenter 2000), and harbors nearly 2000 YSOs within a few parsecs (Hillenbrand \& Hartmann 1998), the cluster core alone containing over 100 YSOs stars within $0.1 \mathrm{pc}$ of the star $\theta^{1}$ Ori C.

Solar system-sized circumstellar disks in the Orion Nebula were first inferred from radio observations of compact ionized regions surrounding young low-mass stars (Churchwell et al. 1987). The Hubble Space Telescope (HST) subsequently provided the most compelling evidence for disks in the spectacular images of extended circumstellar material surrounding young low-mass stars in the core of the Orion Nebula. Over half of the 300 YSO observed in the HST images were classified

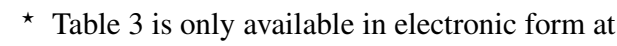
http://www.edpsciences.org as proplyds (PROtoPLanetarY DiskS) (O'Dell et al. 1993; O’Dell \& Wen 1994; O’Dell \& Wong 1996; McCaughrean \& O'Dell 1996; Bally et al. 1998a, 2000), flattened circumstellar clouds of dust and gas surrounding young stars rendered visible through inclusion in or proximity to an $\mathrm{H}$ II region. The intense UV radiation fields of the massive OB stars heat the disk surface, drive mass-loss and produce bright ionization fronts due to the emission-line radiation arising from the outer parts of the proplyds. Most of them are comet-shaped ionized envelopes pointing directly away from the brightest OB stars and are believed to contain evaporating circumstellar disks (McCaughrean et al. 1998; Johnstone et al. 1998). The principal sources of ionizing radiation in the region are the O6, $45 M_{\odot} \operatorname{star} \theta^{1}$ Orionis $\mathrm{C}$, the brightest of the Trapezium compact group of 4 high-mass OB stars, and the O9.5, $25 M_{\odot}$ star $\theta^{2}$ Orionis A located several arcminutes to the south at a distance of $0.3 \mathrm{pc}$ from $\theta^{1}$ Ori C. Dusty disks are seen either as dark silhouettes against the bright background nebular light - the pure silhouettes - (McCaughrean \& O'Dell 1996; Bally et al. 2000) or embedded in the light from their own ionization fronts - the embedded silhouettes (Bally et al. 2000). Proplyds display a variety of forms. The ones closer to $\theta^{1}$ Ori $\mathrm{C}$ have bright cusps in optical emission lines $\left(\mathrm{H}_{\alpha}\right.$, [O III], [N II]) facing $\theta^{1}$ Ori $\mathrm{C}$ and "tails" extending from the ends of the cusps. The farthest have curved and close boundaries that include the entire circumstellar cloud. In the fields of the inner portion of the Orion Nebula - a region that contains more than 300 YSO of the nearly 2000 members of the extended 
Trapezium cluster - imaged with the HST, 161 proplyds were identified, of which 15 are pure silhouettes and 146 are bright droplets surrounded by ionization fronts (IF).

O’Dell (2001a) examined WFPC2/HST parallel planned observations in the outer portions of the Orion Nebula and near the center of its companion H II region M43 located several arcminutes north of the Trapezium and powered by a single B0 spectral type star, NU Ori. His discovery of three new bright proplyds (093-822; 307-1807; 332-1605) and one pure sillhouette proplyd (321-602) is an important proof that additional young stars, disks and jets remain to be revealed in the outer parts of the Orion Nebula. Subsequently, Smith et al. (2005), as a result of an $\mathrm{H}_{\alpha}$ survey with the HST Wide Field Camera of the Advanced Camera for Surveys (ACS/WFC), discovered 10 new silhouette disks in the outskirts of the Orion Nebula and in its neighboring region M 43: 6 pure silhouettes - 053717, 110-3035, 141-1952, 280-1720, 347-1535, 216-0939and 4 embedded silhouettes - 132-042, 124-132, 253-1536, 181-826 (this one discussed in Bally et al. 2005). These new disks exhibit extended emission from bipolar reflection nebulae and microjets due to a fainter background in the less intense radiation fields regions far from the Trapezium core. Up to date publications, 171 proplyds were imaged with the Hubble Space Telescope (HST) in the Orion Nebula and M 43: 150 bright cusps and 21 pure silhouettes.

In this paper we analyze available HST-WFPC2 images to measure the diameters of 149 proplyds (14 are pure silhouettes and 135 are bright proplyds surrounded by ionization fronts) and derive the basic statistics of this population. These statistics can provide important constraints to models of protoplanetary disk evolution and planet formation in young stellar clusters. The observations are described in Sect. 2 and the results presented in Sect. 3. The results are discussed in Sect. 4 and the main conclusions are summarized in Sect. 5.

\section{Observations}

To perform the diameter measurements we selected from the available Hubble Space Telescope (HST) images of the Orion Nebula (Bally et al. 2000) ${ }^{1}$ the one that provided the best contrast between the disk and the background nebula light. The available images are mosaic images from data acquired through filters F673N, F658N, F656N, F631N, F547N and $F 502 N$, under the general observer (GO) programs, GO 5085 (O’Dell \& Wong 1996) and GO 5469 (Bally et al. 1998a; O'Dell 1998). After quantitative comparisons we selected the $\mathrm{H}_{\alpha}$ image since it provided the best contrast and had the best signal-to-noise. The selected $\mathrm{H}_{\alpha}$ image covers an area in the sky of $7.8 \times 8.3 \mathrm{arcmin}^{2}$ and has a resolution of about 0.'1. World Coordinate System (WCS) coordinates were inserted using IRAF ${ }^{2}$ scripts and the images were searched for

\footnotetext{
1 The HST mosaicked "master" images of the Orion Nebula are accessible at

http://casa. colorado.edu/ bally/HST/HST/master/

${ }^{2}$ IRAF is distributed by the National Optical Astronomy Observatory, which is operated by the Association of Universities for Research in Astronomy, Inc., under cooperative agreement with the National Science Foundation.
}

circumstellar disks seen only in silhouette, bright proplyds with dark disks, and bright proplyds without visible disks. The names of the objects discussed in this paper use the coordinatebased system introduced by O'Dell \& Wen (1994), where the numbers are truncated positions (in the equinox J2000.0 FK5 reference system) rounded off to 0.1 of right ascension and $1^{\prime \prime}$ in declination. The first three coordinates correspond to the right ascension $(\mathrm{J} 2000.0)$ given to $0^{\mathrm{s}} .1$, with $5^{\mathrm{h}} 35^{\mathrm{m}}$ subtracted and the decimal indicator dropped. The last three digits correspond to the declination (J2000.0) with $5^{\circ} 20^{\prime}$ added. For example, 131-046 has a right ascension of $5^{\mathrm{h}} 35^{\mathrm{m}} 13^{\mathrm{s}} .1$ and a declination of $-5^{\circ} 20^{\prime} 46^{\prime \prime}$. When the object lies north of $-5^{\circ} 20^{\prime}$, a four digit declination indicator is used, like in 121-1925.

For the image analyzed in this paper the typical errors in the positions are about 0.5 pixels or $0 . ' 05$. For a distance of $450 \mathrm{pc}$ to the Orion Nebula, the physical resolution of the HST image is 0 .' 15 or $\sim 67.5$ AU. ( $\sim 1.5$ pixel/FWHM; 1 pixel corresponds to $\sim 0$.' 1 or 45 AU). Bally et al. (2000) analyzed dithered images obtained with the WFPC2 in the HST Cycle 6 under program GO 6603. The dithered images were combined into final images using the drizzling technique resulting in individual wide-field frames with $20 \%$ better resolution than the nondrizzled image analyzed in this paper. This has an impact on the measurements of the proplyds' sizes, i.e., the diameters measured in this paper are, on average, $20 \%$ greater than the diameters listed in Bally et al. (2000).

\section{Results}

\subsection{The sample}

We assume circumstellar disks to be essentially circular structures (typical disk thickness, $\sim 10 \%$ of diameter, is negligible when compared to the precision of our measurements, see Sect. 3.2). Projections of a circular disk at any random orientation always corresponds to an ellipse, exception given to the perfect edge-on case. The diameter of the major axis of such an ellipse is always equal to the diameter of the circular disk seen in projection. Hence, a direct measurement of the major axis of an observed ellipse is a direct measurement of the disk diameter.

The initial sample consisted of 161 sources (see Table 3, available in electronic form), all listed in previous papers (O'Dell et al. 1993; O’Dell \& Wen 1994; O’Dell \& Wong 1996; McCaughrean \& O’Dell 1996; Bally et al. 2000) but only 144 of them were identified in our analysis (14 were not found, 2 were not included and 1 was out of the image). We found an object which was clearly proplyd-like but its coordinates did not correspond to any object in previous catalogs. We named it 266-557 using O'Dell's coordinate-based system and added it to the sample. For the 14 proplyds not found in our analysis, 2 are silhouettes (132-1832; 172-028) and 12 are bright proplyds and they are, either indistinguishable from the background $(064-705 ; 153-1902 ; 158-425 ; 166-519 ; 171-$ 315 ; 174-400; 179-536; 205-421; 208-122; 215-317; 221$433)$, or very close to a bright star (163-322). We suggest that some of their coordinates can be possibly wrong. The proplyds 168-326NW and 168-326SE, listed as two different objects in 
previous papers, were not resolved in the image as two objects, so they were not included in the sample. From the 15 silhouettes discovered until and listed in the paper Bally et al. (2000), the silhouette 294-606 appears out of the field of view in the $\mathrm{H}_{\alpha}$ mosaic image we used and, therefore, was not considered in the sample. The proplyds 132-1832, 172-028, 171-315 and 205-421 were measured directly from their published images in the papers O'Dell \& Wen (1994) and Bally et al. (2000), resulting in a final sample of 149 proplyds where 14 are pure silhouettes and 135 are bright proplyds. Table 3, available in electronic form, lists the observed properties of all the 162 externally illuminated YSOs considered in this paper, measured coordinates (RA and Dec), disk and ionization front diameters in pixel and $\mathrm{AU}$ (measured in $\mathrm{H}_{\alpha}$ emission), projected distances to $\theta^{1}$ Ori $\mathrm{C}$ and comments. See discussion on the procedure followed in the next section.

O'Dell (2001a) and Smith et al. (2005) proplyds were not included in our sample because they lie farther north from the region covered by our $\mathrm{H}_{\alpha}$ image.

\subsection{Procedure}

The measuring procedure can be summarized as the following: for the dark silhouettes the diameter in pixel was considered to be the diameter of an ellipse fitted to the $\sim 10 \%$ contour below the background intensity. Because of the highly variable background in the Orion Nebula, the considered value for the intensity, is an average $(I \max +I \min ) / 2$, of the immediate background surrounding the disk. For the bright proplyds, within the variety of dimensions that can be measured, the most useful and practical is the "chord diameter", meaning the distance between the tips of the cusps of the proplyds as described by O'Dell (1998). This can be determined more accurately than any other dimension, such as the size along the direction from the ionizing star to the cusp, and should not vary with different spatial orientations. But, the uncertainty of the chord diameter is primarily determined by the judgment involved in where to define the cusp boundary and amounts 1 to 2 pixels. To overcome this problem, we defined the cusp boundary as a circle fitted to the contour of plus $\sim 30 \%$ the average background intensity. The "chord diameter" is then the diameter of this circle with an uncertainty of 0.5 pixel or $22.5 \mathrm{AU}$, the error associated with the measurement only. Unfortunately, because of the highly variable background intensity, the contrast between proplyds/silhouettes and the background is sometimes low. In our sample of 135 bright proplyds, 37 objects, or $27 \%$ of the total sample, were not bright enough so they were fitted to a contour of $+10 \%$ the average background intensity. At the same time, although clearly detected, 4 silhouettes were not dark enough and were measured to plus a few percent.

\subsection{Do the proplyd diameters vary with distance from $\theta^{1}$ Orionis $C$ ?}

The observational establishment of the proplyds as a well defined class of objects has led to several attempts to theoretically model them (Henney et al. 1996; Johnstone et al. 1998;
Henney \& Arthur 1998; Störzer \& Hollenbach 1999; Richling $\&$ Yorke 2000). In this section we will introduce the most important aspects of proplyd photoevaporation models to stress that a correlation between proplyd diameter and distance from the ionizing source is expected in theory.

The diameter of the hydrogen ionization fronts (bright proplyds) is a complex function dominated by the incident UV photon flux or true distance to the illuminating source and the disk radius. The appearance of the proplyds can be explained by the interaction of EUV - extreme-ultraviolet (Lyman continuum; $h v>13.6 \mathrm{eV}$ ) and FUV - far-ultraviolet $(6 \mathrm{eV}<h v<13.6 \mathrm{eV})$ radiation from an external source with a circumstellar disk. The FUV photons are absorbed mainly by dust and penetrate much deeper into the disk than the EUV photons. At relative high column densities they dissociate molecules, heat the material impenetrable to the EUV photons and initiate a neutral flow away from the disk. This "photon-dominated region" or PDR is encased within an hydrogen IF where the outflowing PDR material can be ionized by the EUV photons. Depending on the ratio $I(\mathrm{UV})=\mathrm{FUV} / \mathrm{EUV}$, the IF can stand off at a considerable distance from the disk surface, appearing as a bright round head in the direction of the illuminating source with an extended "cometary" tail of lower emissivity in the far side of the disk. Johnstone et al. (1998), presented analytical and numerical models of the structure of the neutral flows. Depending on the disk radius, the $I(\mathrm{UV})=\mathrm{FUV} / \mathrm{EUV}$ ratio and the column of neutral gas in the PDR, the neutral flow can be either FUV-dominated or EUV-dominated. For a given disk outer radius, as the distance to the ionization source increases, the EUV flux declines, I(UV) increases and the radius of the IF is expected to increase. The most rapid disk erosion occurs for large systems close to the Trapezium. Störzer \& Hollenbach (1999) improved the previous Johnstone et al. (1998) model including the results of both equilibrium and non-equilibrium photo-dissociation region (PDR) codes to calculate the column density and temperature inside the PDR. They determined under which circumstances FUV-dominated flows are possible and explained the observed IF diameters of the Orion proplyds by the FUVdominated flows. These FUV-dominated flows are extended with IF radius $r_{\mathrm{IF}} \propto d_{\theta^{1} \text { OriC }}^{2 / 3}$ and typically, $r_{\mathrm{IF}} \gtrsim 2 r_{\mathrm{d}}$, for a distance of $0.01-0.3$ pc from $\theta^{1}$ Ori C. The mass-loss rate is proportional to the disk radius and not so dependent on the distance to $\theta^{1}$ Ori C. Outside this region, EUV photons dominate the photoevaporation, the IF is closer to the disk surface, $r_{\mathrm{IF}} \lesssim 2 r_{\mathrm{d}}$, and the mass-loss rate is proportional to $d_{\theta^{1} \text { OriC }}^{-1}$ and $r_{\mathrm{d}}^{3 / 2}$.

Is there any observed systematic variation of the proplyds diameter with the distance to the main ionizing stars?

Figure 1 presents the IF chord diameter as a function of the projected distance to $\theta^{1}$ Ori $\mathrm{C}$ for the 135 bright proplyds sample. The different symbols indicate the main ionizing stars and, for each proplyd, they were determined by the relative orientation of the bright cusps and tails. For the not so clear cases, we drew a line in our image connecting the ionizing stars and the center of the cusp and compared them with the proplyd's tail-cusp direction. The result of this procedure was that there are 105 proplyds primarily ionized by $\theta^{1}$ Ori $\mathrm{C}$, 


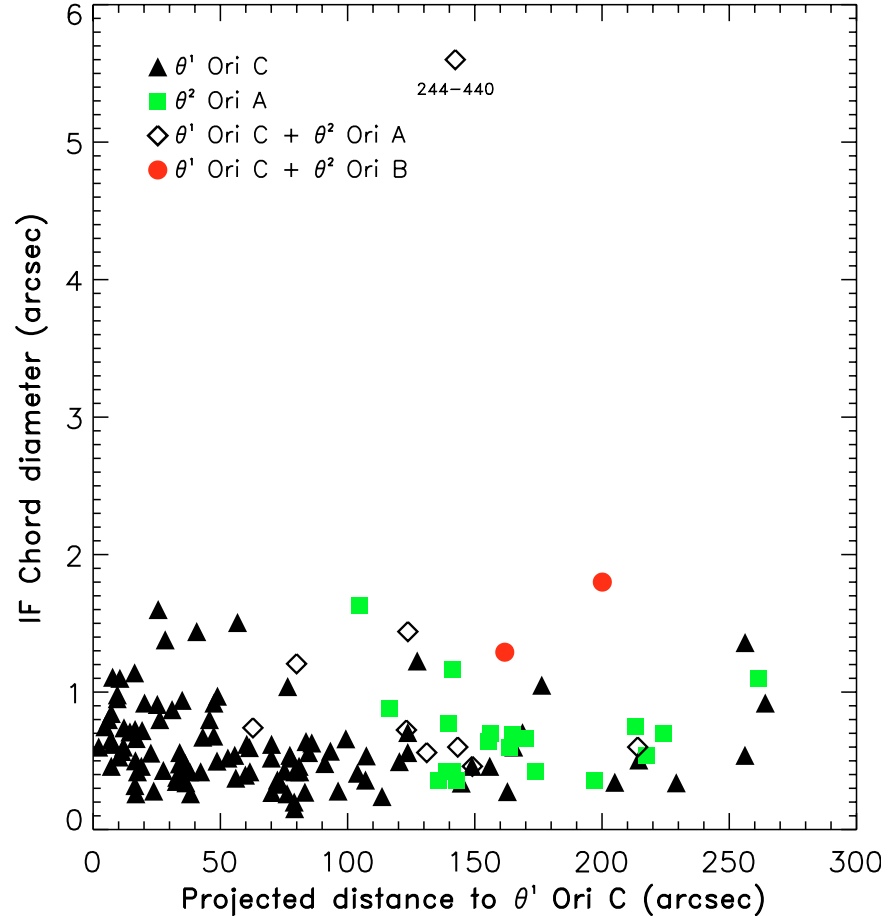

Fig. 1. Ionization front (IF) "chord diameter" (diameter of a circle fitted to the contour of plus $\sim 30 \%$ the average background intensity), as a function of the projected distance to the main ionizing star in the Trapezium cluster, $\theta^{1}$ Ori $\mathrm{C}$. The different symbols represent the proplyds that are ionized primarily by the stars indicated in the plot legend. For example, the largest bright proplyd $244-440$ is being ionized by both $\theta^{1}$ Ori C and $\theta^{2}$ Ori A. There are 2 proplyds (252-457; 282-458) being ionized by both $\theta^{2}$ Ori $\mathrm{B}$, the bright star in the east direction from $\theta^{2}$ Ori $\mathrm{A}$, and $\theta^{1}$ Ori C.

19 by $\theta^{2}$ Ori A, 9 by both $\theta^{1}$ Ori C and $\theta^{2}$ Ori A (like 244-440) and 2 ionized by $\theta^{1}$ Ori $\mathrm{C}$ and $\theta^{2}$ Ori B (252-457 and 282458 ). Figure 1 clearly shows no obvious correlation between the "chord diameter" and the projected distance to the ionizing Trapezium OB stars. Assuming a random distribution of disk sizes across the cluster, this result hints at poor correlation between IF size and distance, unlike theoretical predictions.

\subsection{Deriving disk diameters from the IF chord diameters}

It is not trivial to infer disk diameters from the bright ionization front diameters. Nevertheless, empirically, one can investigate if there is a typical diameter ratio $R(R=$ embedded disk diameter/ionization front diameter) since we have in the sample bright proplyds with clearly resolved embedded disks. We identified 10 such cases (072-135; 141-520; 143-552; $163-222$; 176-543; 181-247; 182-413; 197-427; 206-446; 244-440), at different projected distances from the ionizing sources, and measured both the diameter of the ionization front cusp and the diameter of the disk, as described in Sect. 3.2. Figure 2 represents the $R$ values for the 10 silhouettes with embedded disks as a function of the disk diameters. The different symbols are related to the different main ionizing stars. We find the average diameter ratio for these 10 sources to

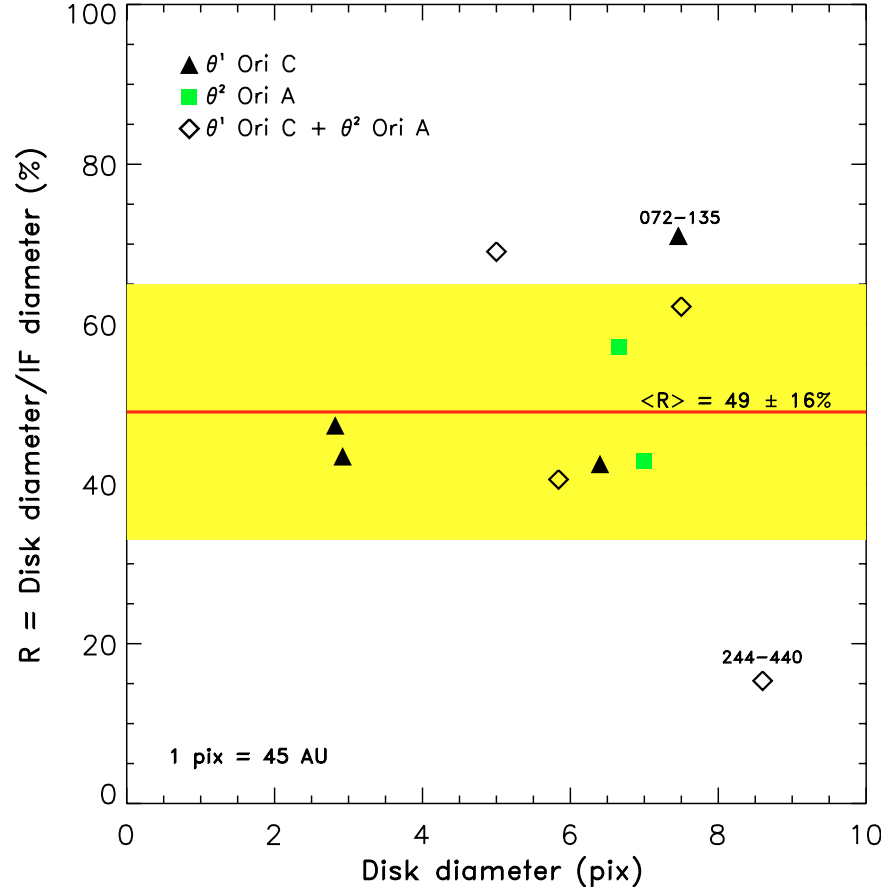

Fig. 2. Diameter ratio, $R=$ disk diameter/ IF diameter, as a function of the disk diameter in pixels. The gray (yellow) band represents the average and dispersion of the sample $(49 \pm 16 \%)$.

be $\langle R\rangle=49 \pm 16 \%$, a value essentially identical to theoretical expectation for a distance of $0.01-0.3 \mathrm{pc}$ from $\theta^{1}$ Ori C (Störzer \& Hollenbach 1999). The observational parameters for these 10 proplyds are listed in Table 1.

Figure 3 is a spatial distribution diagram of the 10 proplyds with embedded silhouette disks. The black filled circles represent the silhouette disks and the colored, unfilled disks represent the ionization fronts. Green is for the proplyds ionized primarily by $\theta^{1}$ Ori C $(072-135 ; 163-222 ; 181-247 ; 182-413)$, blue for the proplyds ionized by $\theta^{2}$ Ori A $(176-543 ; 206-446)$ and red for proplyds ionized by both stars (141-520; 143-522; 197-427; 244-440). The circle diameters are proportional to the disk and IF diameters.

There is a fairly large dispersion in the ratio $R=$ disk diameter/IF diameter, as showed by Fig. 2, but it is not clear that $R$ is correlated with the projected distance, or true distance, to $\theta^{1}$ Ori C (see Fig. 3). For approximately the same proplyd disk diameter there are examples of IFs very close to the disk and IFs that stand at a considerable distances from the disk. There is also no apparent increase of the IF's diameters with the projected distance to $\theta^{1}$ Ori C (Fig. 1). The proplyds 244-440 $(R=15 \%)$ and $072-135(R=71 \%)$ illustrate the two opposite "extreme" cases. $244-440$ is localized at a distance of 142 ". 3 from $\theta^{1}$ Ori $\mathrm{C}$ and 29.2 from $\theta^{2}$ Ori $\mathrm{A}$ and it has the larger IF observed in the Trapezium with a diameter of $2520 \mathrm{AU}$ or 5 .' 6 . One of the reasons could be because it is being ionized by both stars as seen from the shape and orientation of its cusp. Nevertheless, its disk is only $387 \mathrm{AU}$ or 0 ' $^{\prime} 86$. We can find enough cases of proplyds that lie in projection far from $\theta^{1}$ Ori C and still have small IF, despite the large diameter of their disks. For example, $072-135$ is positioned at a distance of 176.3 or 
Table 1. Properties of the 10 proplyds with embedded silhouette disks.

\begin{tabular}{|c|c|c|c|c|c|c|c|c|c|}
\hline \multirow[t]{2}{*}{ Name } & \multicolumn{2}{|c|}{ IF diameter ${ }^{a}$} & \multicolumn{2}{|c|}{ Disk diameter $^{a}$} & \multirow{2}{*}{$\begin{array}{l}R \\
\%\end{array}$} & \multicolumn{2}{|c|}{$d_{\text {proj }} \theta^{1}$ OriC } & \multicolumn{2}{|c|}{$d_{\text {proj }} \theta^{2}$ OriA } \\
\hline & (pix) & (AU) & (pix) & $(\mathrm{AU})$ & & $\left({ }^{\prime \prime}\right)$ & (pc) & $\left({ }^{\prime \prime}\right)$ & (pc) \\
\hline $072-135$ & $10.5(30 \%)$ & 472 & 7.5 & 336 & 71 & 176.3 & 0.38 & 311.3 & 0.68 \\
\hline $141-520$ & $7.2(10 \%)$ & 326 & 5.0 & 225 & 69 & 123.1 & 0.27 & 134.1 & 0.29 \\
\hline $143-522$ & $14.4(10 \%)$ & 648 & 5.8 & 236 & 40 & 123.7 & 0.27 & 130.4 & 0.28 \\
\hline $163-222$ & $6.0(10 \%)$ & 268 & 2.8 & 127 & 47 & 61.4 & 0.13 & 185.2 & 0.40 \\
\hline $176-543$ & $11.7(10 \%)$ & 525 & 6.7 & 300 & 57 & 141.3 & 0.31 & 91.8 & 0.20 \\
\hline $181-247$ & $6.7(10 \%)$ & 302 & 2.9 & 131 & 43 & 43.1 & 0.09 & 149.5 & 0.33 \\
\hline $182-413$ & $15.1(30 \%)$ & 678 & 6.4 & 288 & 42 & 56.7 & 0.12 & 83.4 & 0.18 \\
\hline $197-427$ & $12.1(30 \%)$ & 543 & 7.5 & 337 & 62 & 79.9 & 0.17 & 57.7 & 0.13 \\
\hline $206-446$ & $16.3(30 \%)$ & 734 & 7.0 & 315 & 43 & 104.6 & 0.23 & 35.9 & 0.08 \\
\hline $244-440$ & $56.0(30 \%)$ & 2520 & 8.6 & 387 & 15 & 142.3 & 0.31 & 29.2 & 0.06 \\
\hline
\end{tabular}

${ }^{a}$ The disk and IF diameters were determined directly from the data and do not account for finite size of the HST PSF.

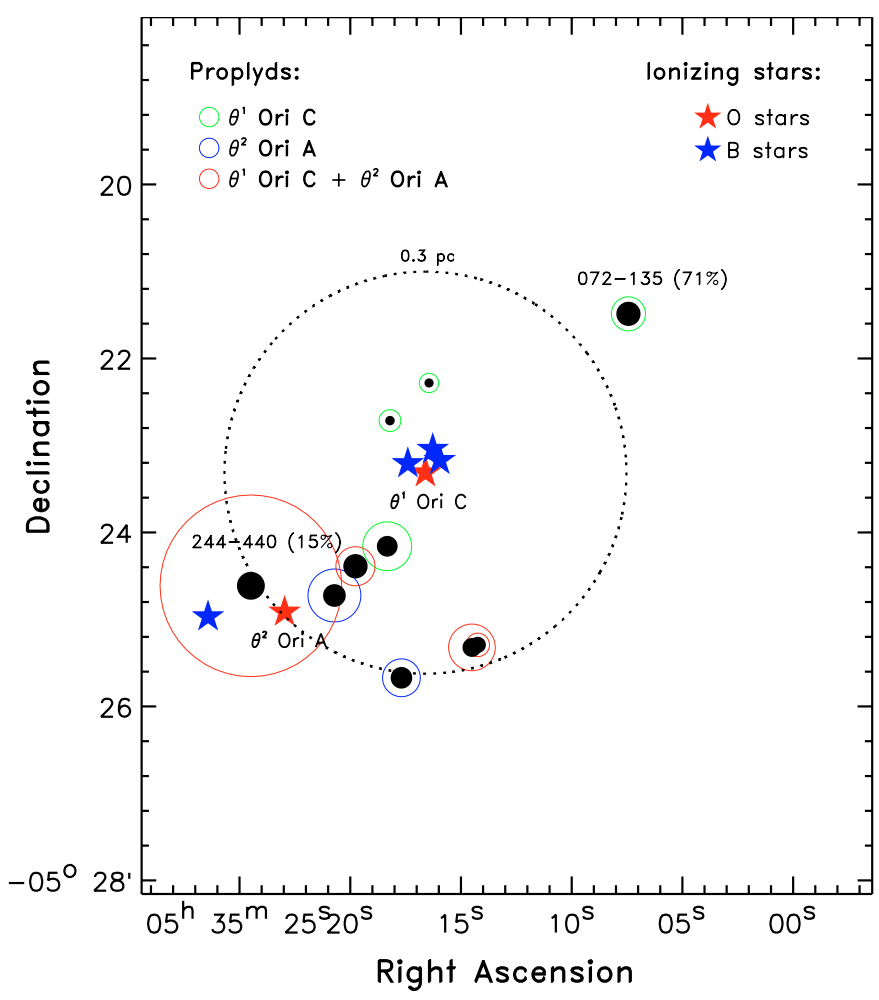

Fig. 3. Spatial distribution of the 10 proplyds with embedded silhouette disks. The black filled circles represent the silhouette disks and the colored, open circles represent the ionization fronts. Green is for the proplyds ionized primarily by $\theta^{1}$ Ori C $(072-135 ; 163-222$; 181-247; 182-413), blue for the proplyds ionized by $\theta^{2}$ Ori A (176-543; 206-446) and red for proplyds ionized by both stars (141-520; $143-522 ; 197-427 ; 244-440)$. The circle diameters are proportional to the true disk and IF diameters.

$0.38 \mathrm{pc}$ in the north-west direction from $\theta^{1}$ Ori $\mathrm{C}$ and has a small IF of $11^{\prime \prime} 05$ with a large disk of 0.75 . At the same time, 176-543, approximately at the same distance from $\theta^{1}$ Ori $\mathrm{C}$ as $244-440$ or 141 '. 3 , has an IF of only 1 .' 17 or $\sim 1 / 5$ of $244-440$ 's chord diameter. Its disk is $00^{\prime} 67$ and $R=57 \%$. In summary, there is no obvious indication for larger proplyds to be located farther from the UV source, even taking into consideration the unknown distant projection correction. In conclusion, it seems reasonable to adopt the average value of $R,\langle R\rangle=49 \pm 16 \%$, from our sample of 10 proplyds distributed at different distances, and use it as a calibrator to compute the disks diameters for the 125 bright proplyds without embedded silhouettes. We find the same general result in the data from previous measurements by O'Dell (1998) and Bally et al. (1998a), discussed in the next section.

\subsection{Comparison with previous work}

In this section we make a detailed comparison of the data presented in this paper and the observational data published in previous papers - Bally et al. (1998a), O’Dell (1998), Johnstone et al. (1998), Störzer \& Hollenbach (1999) and Bally et al. (2000) (see Table 2).

For the subsample of proplyds listed in these papers, and common to our sample, an identical analysis was performed. Because the assumed distances to the clusters used in the different papers are not always the same (we use $d_{\text {Orion }}=450 \mathrm{pc}$ through out this paper) we scaled all results to the same distance and only then compared them. To compare the different subsamples of proplyds with embedded disks we proceeded as follows: we computed the diameter ratio $R$ for each proplyd, the average diameter ratio $\langle R\rangle$ for all the subsample of proplyds, and the linear correlation coefficient $r$ between the disk and IF diameters and the projected distances to the main ionizing $\operatorname{stars}^{3}$.

Bally et al. (1998a) observed four fields near the Trapezium with the Planetary Camera (PC), the 0.'05 angular resolution portion of the WFPC2/HST, on March 1995. Observations were obtained through narrow-band filters centered on the [S II], [N II], $\mathrm{H}_{\alpha}$, [O I] and [O III] lines and in an emissionline-free continuum channel. They present the dimensions of

${ }^{3}$ The correlation coefficient $r$ is a measure of the linear association between variables. The strength of this association is sometimes expressed as the square of the correlation coefficient. The resulting statistic is known as variance explained. For example, a correlation of 0.5 means that $(0.5)^{2}$ or $25 \%$ of the variance in $Y$ is "explained" or predicted by the $X$ variable. This parameter is used throughout this paper. 
Table 2. Orion proplyds observational parameters: comparison with previous papers.

\begin{tabular}{|c|c|c|c|c|c|c|c|c|c|}
\hline \multirow[t]{2}{*}{ Paper } & \multicolumn{2}{|c|}{ Total } & \multicolumn{2}{|c|}{ Common } & \multicolumn{2}{|c|}{ Max. $d_{\text {proj }} \theta^{1}$ OriC } & \multirow{2}{*}{$\begin{array}{l}\langle R\rangle \\
(\%)\end{array}$} & \multirow{2}{*}{$\begin{array}{r}r_{\mathrm{IF}} \\
(\%)\end{array}$} & \multirow{2}{*}{$\begin{array}{l}r_{\text {disk }} \\
(\%)\end{array}$} \\
\hline & IF & disks & IF & disks & $\left({ }^{\prime \prime}\right)$ & (pc) & & & \\
\hline B98 $a^{a}$ & 40 & 21 & 34 & 19 & 70.0 & $0.15(184-427)$ & $43 \pm 18$ & 30 & 14 \\
\hline O98 $8^{b}$ & 22 & - & 20 & - & 70.0 & $0.15(184-427)$ & - & 22 & - \\
\hline JHB9 $8^{c}$ & 41 & 15 & 30 & 15 & 70.0 & $0.15(184-427)$ & $48 \pm 22$ & 42 & 7 \\
\hline $\mathrm{SH} 9^{d}$ & 10 & 10 & 10 & 10 & 56.7 & $0.12(182-413)$ & $48 \pm 20$ & 55 & 70 \\
\hline $\mathrm{B} 00^{e}$ & 24 & 24 & 18 & 18 & 176.3 & $0.38(072-135)$ & $44 \pm 17$ & 2 & 8 \\
\hline
\end{tabular}

${ }^{a}$ Bally et al. (1998a); ${ }^{b}$ O’ Dell (1998); ${ }^{c}$ Johnstone et al. (1998); ${ }^{d}$ Störzer \& Hollenbach (1999); ${ }^{e}$ Bally et al. (2000).

This table refers only to the sample of bright proplyds listed in previous papers and deliberately excludes the pure silhouettes. The second column refers to the total number of bright proplyds (here IF) and bright proplyds with embedded disks (here disks) listed in the papers. The third column refers to the subsample of objects that are common to our sample. $\langle R\rangle$ is the diameter ratio computed for the common sample of embedded disks. The maximum projected distances listed here are the ones measured in this paper assuming 1 pix $\sim 0$." $1=45$ AU and $d_{\text {Orion }}=450 \mathrm{pc}$; the differences between the different papers were negligible. The linear correlation coefficients $r_{\mathrm{IF}}$ and $r_{\mathrm{disk}}$ are represented by its square times $100 \%$. Bally et al. (2000) makes reference to many other proplyds but, we just consider the $24 \mathrm{H}_{\alpha}$ emission bright proplyds with dark disks embedded.

the various emission and opaque components, and the peak surface brightness of the objects in each emission line, for 43 Trapezium proplyds, of which 40 are bright (21 with dark disks embedded) and 3 are pure silhouettes. From these, 38 are common to our sample ( 35 bright proplyds +3 pure silhouettes) and 19 are bright proplyds with dark embedded disks. For the maximum projected distance to $\theta^{1}$ Ori $\mathrm{C}$ in the sample (184-427 at $70^{\prime \prime}$ ), the average diameter ratio $\langle R\rangle$ computed for the 19 proplyds is $43 \pm 18 \%$. Bally et al. (1998a) claims that, when both silhouette and IF are seen, the ratio of the semimajor axis of the silhouettes divided by the IF radius ranges from 0.25 to 0.67 . This is also confirmed in our analysis. The values of $R$ show a large dispersion but are in that range, with exceptions given to $158-327,160-353$ and 166-316. The authors argue that there is a loose correlation between the cusp radius and the projected distance to $\theta^{1}$ Ori $\mathrm{C}$, with the cusp radius increasing as $r(d) \propto d^{\alpha}$ with $\alpha=0.5-0.8$, where $d$ is the projected distance from $\theta^{1}$ Ori C. For the 34 bright proplyds common to our sample, we find $\alpha=0.46$. The linear correlation coefficient between the two variables is 0.54 or $\sim 30 \%$, meaning a loose correlation for this specific sample of proplyds.

O'Dell (1998) presents a set of 22 bright proplyds observed in the Bally's GO program 5469 with the HST/PC in 1995, of which 20 are common to our sample and 19 to Bally et al. (1998a). The author found a very loose correlation of the proplyd cusp diameter with the distance from $\theta^{1}$ Ori $\mathrm{C}$, for a maximum projected distance in his sample of $70^{\prime \prime}(184-427)$ or $\sim 0.15$ pc. We computed the linear correlation coefficient for the 20 common sources and found a value of 0.47 or $22 \%$, indicator of a minor correlation between the variables.

Johnstone et al. (1998) compare their model results with HST observations from Bally et al. (1998a). For 15 proplyds disks, for a maximum projected distance to $\theta^{1}$ Ori $\mathrm{C}$ of $70^{\prime \prime}$ (184-427), the average diameter ratio $\langle R\rangle$ equals $48 \pm 22 \%$. The correlation coefficient between disk diameters and projected distance to $\theta^{1}$ Ori $\mathrm{C}$ is 0.26 or $7 \%$. This paper presents a sample of 41 proplyds of which 30 (27 common to our sample) were measured with the HST Planetary Camera and 11 (3 common to our sample) with the Wide Field Camera.
The total 30 bright proplyds IF diameters are correlated with the distance to $\theta^{1}$ Ori $\mathrm{C}$ by $42 \%$ or $r=0.64$, indicating a large correlation between them.

Störzer \& Hollenbach (1999) used the best 10 measured disks reported by Johnstone et al. (1998), in which both disk and IF diameters could be observed (Bally et al. 1998a). For a maximum projected distance of 56."7 (182-413 or HST10), $\langle R\rangle=48 \pm 20 \%$. There is a very high correlation between both disk and IF diameters and the projected distance to $\theta^{1}$ Ori $\mathrm{C}$, $r_{\mathrm{IF}}=55 \%$ and $r_{\text {disk }}=70 \%$. But, we have to keep in mind that this is a very particular sample.

The last test was performed in the data from Bally et al. (2000). Although, the only diameters listed are for the 15 pure silhouettes, they published images of all the embedded disks seen against the bright ionization fronts: 24 disks in $\mathrm{H} \alpha$, 16 disks in [O III] and 2 disks in [N II]. The IF, and disk diameters of 17 proplyds (disks boundaries are not clear in all pictures) were measured directly from the published $\mathrm{H} \alpha$ images. The proplyd 141-520 measures were taken from the [N II] image, increasing the sample, from Bally et al. (2000) paper, to 18 bright proplyds. There is no significant correlation between the disk and IF diameters and the projected distance to $\theta^{1}$ Ori C. For this sample: $r_{\mathrm{IF}}=2 \%$ and $r_{\mathrm{disk}}=8 \%$, for a maximum projected distance of 176 '. $3(072-135)$ or $0.38 \mathrm{pc}$. The average diameter ratio is $\langle R\rangle=44 \pm 17 \%$ and the IF diameters are, on average, $1.2 \pm 0.2$ times smaller than the ones in this paper, in agreement with the $20 \%$ increased resolution of Bally et al. (2000) drizzled images. For our sample of 135 bright proplyds, and separating them in subsamples accordingly with the main ionizing $\mathrm{O}$ star, we computed the linear correlation coefficient between the disk and IF diameters and the projected distance to the main ionizing source. The first subsample is composed of 105 bright proplyds which are being ionized primarily by $\theta^{1}$ Ori $\mathrm{C}$, and we found that there is no correlation between the two variables for a maximum projected distance of $264^{\prime \prime}$ (005-514) (Fig. 1). When we consider just the proplyds which are at a distance less than 0.3 pc (Störzer \& Hollenbach 1999), the correlation is low $(-0.26$ or $7 \%)$. For the 19 proplyds ionized by $\theta^{2}$ Ori $\mathrm{A}$, for a maximum projected distance of $151^{\prime \prime}$ 
(224-728), we get a week correlation of $13 \%$ for IF diameter with the projected distance to $\theta^{2}$ Ori A, even for distances less than $0.3 \mathrm{pc}$. The disks show a higher correlation of $18 \%$.

In summary, a dispersion of $\mathrm{R}$ for the Trapezium proplyds is found by all the authors for the previous papers and, in all of them as well, there is no obvious correlation between the IF "chord diameter" and the projected distance to the Trapezium O stars. Moreover, and within the errors, all previous papers agree on the value of $\langle R\rangle$. It seems reasonable to assume an average value for the diameter ratio disk diameter/ IF diameter and use it as a "calibrator" to infer the disks diameters for the 125 bright proplyds without visible disks. The rms in $\langle R\rangle$ will be used to estimate the error in this approximation.

\subsection{Caveats}

Selection effects make the detection of the disks difficult. Dark silhouettes can be easily overwhelmed by the unknown lineof-sight contribution from the nebula or from young stars at their centers. This is especially true for small ( $<2$ WFPC2 pixels, or $90 \mathrm{AU}$ ) disks. On the other hand, small high surface brightness proplyds near $\theta^{1}$ Ori $\mathrm{C}$ are hard to distinguish from point sources. In general, the location of a proplyd with respect to the illuminating stars strongly influences the visibility of embedded circumstellar disks. The procedure for defining disks and proplyds boundaries assumed in this paper is systematic and precise but dependent on the surrounding object nebular intensity, which is highly variable. Nevertheless, this is still the best possible size measurement procedure. Previous papers do not define the measuring procedure and proplyds' sizes are even more dependent on personal judgment. Estimating the completeness of our sample is a difficult task. Still, given that the spatial resolution of the WFPC2 image is 67.5 AU, we can crudely estimate that to a visual extinction limit of a few magnitudes of $A_{\mathrm{V}}$ our sample should be essentially complete for disk diameters larger than $\sim 100-150$ AU.

\subsection{Size distribution of disks}

In Fig. 4, we present the spatial diameter distribution of all the 149 disks in our sample of the Trapezium cluster. The "star" symbols represent the 6 brightest Trapezium OB stars. The filled circles indicate the positions of the 135 bright proplyds' disks; gray (green) for the 10 proplyds with embedded silhouette disks and black for the remain 125 from which the disk diameter is $49 \%$ of the correspondent IF diameter. The unfilled circles represent the positions of the 14 pure silhouettes. The diameters of the circles are proportional to the disk diameters they represent. The pure silhouettes are the largest disks that lie at relatively large projected distances from the Trapezium. Two proplyds seem to fall off the size distribution i) 114-426, with a diameter of $1242 \mathrm{AU}$, is by far the largest and ii) 218-354, with a diameter of $675 \mathrm{AU}$. The dashed circle indicates a radial distance of $0.3 \mathrm{pc}$ from $\theta^{1}$ Ori $\mathrm{C}$ and marks the limiting border of the FUV-dominated region of the Störzer \& Hollenbach (1999) photoevaporation model. It is also the distance between the two ionizing stars, $\theta^{1}$ Ori $\mathrm{C}$ and $\theta^{2}$ Ori A, with the EUV

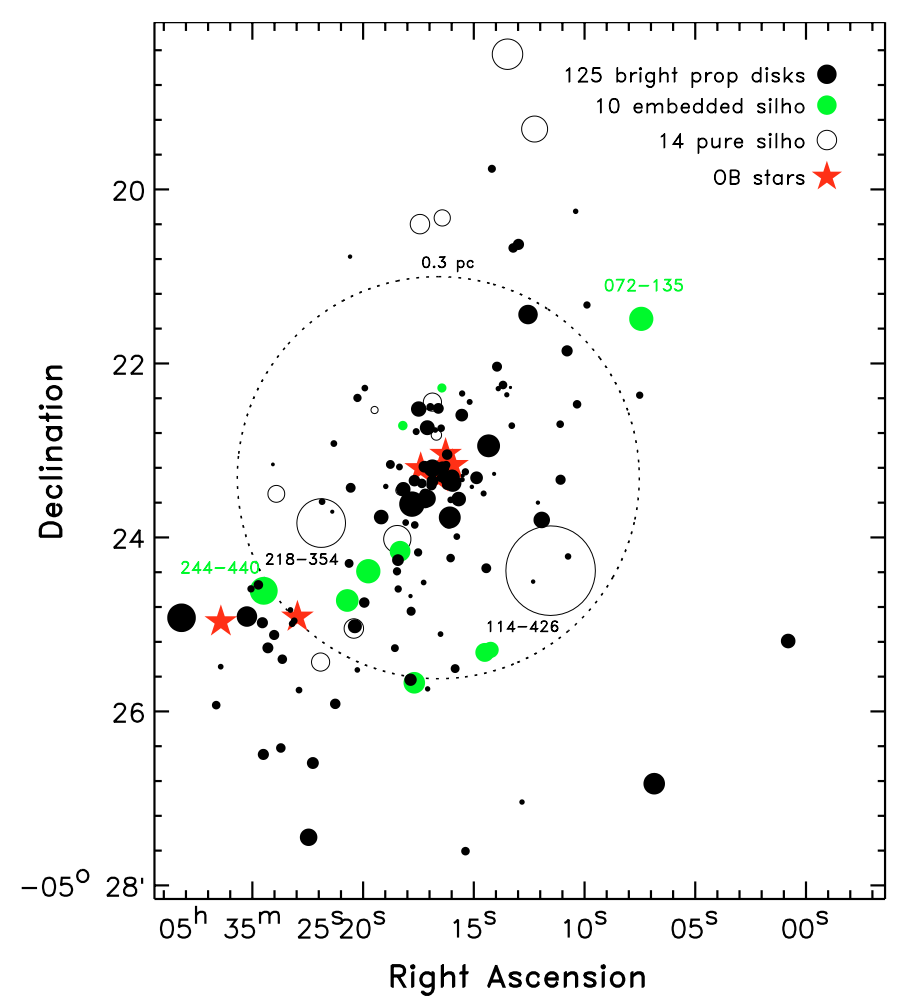

Fig. 4. Spatial distribution of total sample of 149 disks in the Trapezium cluster. The "star" symbols represent the 6 brightest OB stars. The filled circles indicate the positions of the 135 bright proplyds' disks; gray (green) for the 10 proplyds with embedded silhouette disks and black for the remaining 125 from which the disk diameter is $49 \%$ of the correspondant IF diameter. The unfilled circles represent the positions of the 14 pure silhouettes. The diameters of the circles are proportional to the disk diameters they represent.

photoionizing luminosity of the former 3-4 times greater than the later (O'Dell 2001b). About $73 \%$ of the proplyds in our sample are inside this region and $60 \%$ at a projected distance less than $0.2 \mathrm{pc}$. The proplyd situated at the largest distance from $\theta^{1}$ Ori $\mathrm{C}$ is $005-514$ at $\sim 264^{\prime \prime}$ or $0.58 \mathrm{pc}$. We do not find a correlation between the disk diameters and the projected distance to $\theta^{1}$ Ori $\mathrm{C}$, as already discussed before.

Figure 5 represents the disk diameter distribution histogram for the total sample of 149 proplyds: 135 bright proplyds, in gray (green), and 14 silhouettes, in black. To select a statistically significant binning for the distribution we used a density kernel estimator (Silverman 1996) that indicated a bin-width of $100 \mathrm{AU}$ at a $90 \%$ confidence limit. The resultant disk diameter distribution seems unimodal, with a tail to the large diameters, suggesting a single population of disks that is well characterized by a power-law distribution.

Figure 6 represents the power-law fit, in a log-log scale, to the sample of 149 disks presented in Fig. 5. The first and incomplete bin (0-100 AU) was deliberately excluded from the power-law fit. We assumed that all the disk diameters (excluding silhouettes) are $49 \pm 16 \%$ of the proplyds' ionization front diameters, based on the 10 well-known cases with resolved embedded disks. The data is well described by a power-law with an exponent of $-1.9 \pm 0.3$. To determine the uncertainty on the power-law fit we performed several tests, shifting the binning 
starting point by 25,50 and $75 \mathrm{AU}$ and the diameter ratio $R$ for the 125 bright proplyds, to $R=33 \%$ and $65 \%$, accounting for the dispersion found in this ratio. The power-law exponent is the average of the 12 coefficients determined in these tests. An obvious and unique deviation to the power-law distribution characterizing our sample is the silhouette 114-426. With a diameter of about 1242 AU this large disk seems to be an exception compared to other proplyd disks in Orion. About 80 to $90 \%$ of Trapezium's young stars and $\sim 65 \%$ of the brown dwarfs show infrared excess emission characteristic of circumstellar disks (Lada et al. 2000; Muench et al. 2001). Assuming that the fraction of disks is not a strong function of depth into the Orion molecular cloud (near-infrared surveys used by Lada et al. 2000; and Muench et al. 2000, are less affected by dust extinction than the $\mathrm{H}_{\alpha} / \mathrm{HST}$ image analyzed in this paper) and given that the HST images reveal, for about the same area of the cluster, that only $\sim 50 \%$ of the sources are associated with extended circumstellar structures, one can infer that a relatively large fraction of sources in the HST images (30 to 40\%) also have disks but they are smaller than the resolution limit of the HST image. Since there were $~ 300$ YSO imaged with the HST then $\sim 85 \%$, or about 255 of them, should have circumstellar disks but we only identify 149 . This means that the other 106 disks are probably too small to have been resolved by the HST. The virtual sample, or corrected sample of disks in the Trapezium, is composed by the 149 disks from our sample plus 106 disks of 50 AU added. The dashed line in Fig. 5 represents the histogram for the virtual sample of 255 circumstellar disks or $85 \%$ of the YSO observed in the Trapezium cluster. The cumulative disk diameter function of the histogram in Fig. 5 is given by Fig. 7 and indicates that 75 to $80 \%$ of disks have diameters smaller than $150 \mathrm{AU}$ (the uncorrected value is about 60\%). This result is not consistent with Rodmann (2002) who finds that $90 \%$ of the disks have diameters smaller than 80 AU (as referenced in Bate et al. 2003). From this cumulative function we can estimate that about $40 \%$ of the disks in the Trapezium have radius larger than $50 \mathrm{AU}$, the Solar System size.

\subsection{Does disk size depend on the parent star mass?}

In Fig. 8 we present disk diameter as a function of central star spectral-type. Our proplyd list was compared with the Lynne Hillenbrand's online working version of Table 1 (last update: September 2003) from Hillenbrand $(1997)^{4}$ The selection criterion consisted in the overlap of the coordinates of the proplys in the two tables with radius less than 2 arcsec.

For the resulting 52 sources in common, we considered the spectral-types derived by Hillenbrand (1997, optical spectroscopy) and Luhman et al. (2000, NIR spectroscopy). Only 43 of the 52 sources had a spectral classification and only 21 of them are classified by Luhman et al. (2000). For the 21 Luhman sources, represented in Fig. 8 as gray (green) squares, we assumed the error to be equal to the uncertainty interval in Luhman's NIR spectral classification. The errors assumed for the 22 Hillenbrand sources are \pm 1 spectral subclass,

\footnotetext{
${ }^{4}$ http://www.astro.caltech.edu/ lah/papers/orion_main.table1.working
}

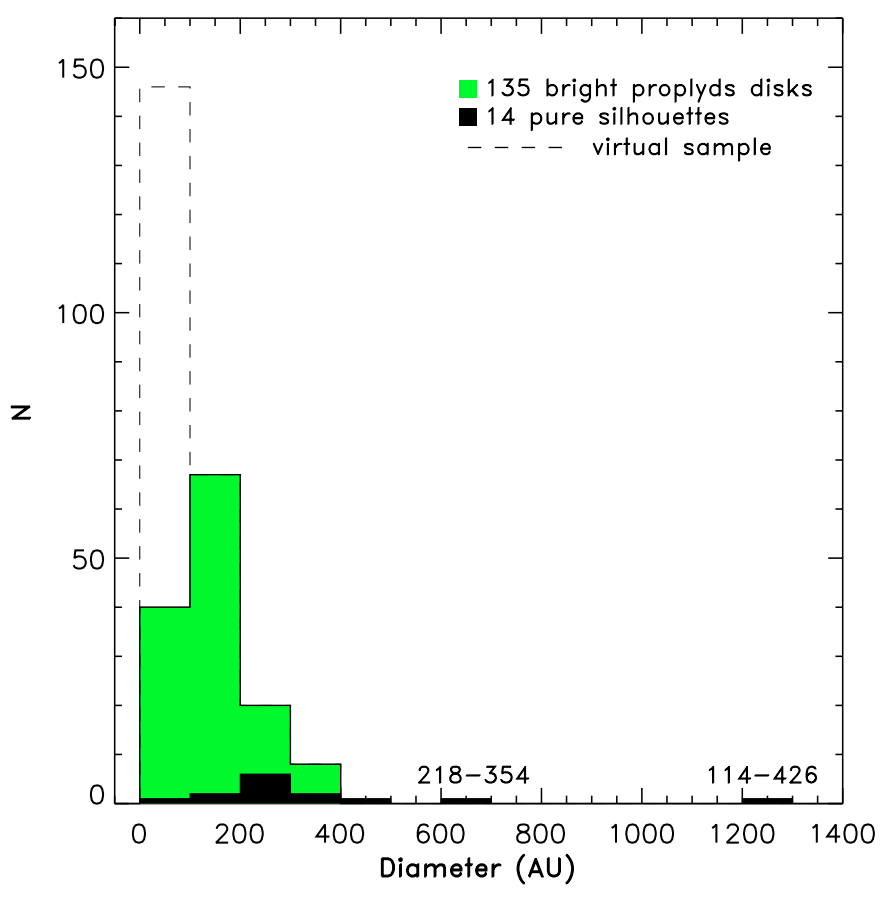

Fig. 5. Disk diameter distribution for the total sample of 149 proplyds. The gray (green) filled histogram represents the 135 bright proplyds disks - 10 dark disks embedded in the bright proplyds' cusps and 125 disks derived from the cusps diameters. The black filled histogram represents the 14 pure silhouettes seen only against the Orion Nebula background. The first bin (0-100 AU) is highly incomplete due to the resolution limit of the data. The virtual sample, in dashed line, was estimated based on the $L$-band excess of Trapezium sources. The large silhouettes 218-354 and 114-426 are indicated.

for spectral types later than $\mathrm{K} 7$, and $\pm 1 / 2$ class for spectral types former than $\mathrm{K} 7$, as described in her paper. They are represented as black triangles in the plot. A systematic error of $45 \mathrm{AU}$ or 1 pixel was considered in the disk diameters.

In order to describe the strength of the linear association between the mass of the star and the diameter of the disk surrounding it, several tests were performed on the data. We calculated the Pearson linear correlation coefficient for all the 43 sources of overlap between our sample and Hillenbrand's catalog. To verify if this linear correlation was or not dependent on the way that the YSOs spectral types were determined, we separated the 43 combined data in two samples: 21 proplyds from Luhman et al. (2000), and 22 proplyds from Hillenbrand (1997). For the combined sample we derive a coefficient of 0.3 indicating a small correlation between the two variables (10\%). However, we get a better correlation for Luhman sources alone (suggesting a significant correlation between diameters and masses) than in Hillenbrand sample where we get a very small correlation. A larger sample or a better understanding of spectral typing differences between optical and NIR should clarify this inconsistency. We conclude that, over the sampled mass range (late $G$ to late $M$ stars) and with the present spectral data, there is not an obvious correlation between disk diameter and stellar mass. 


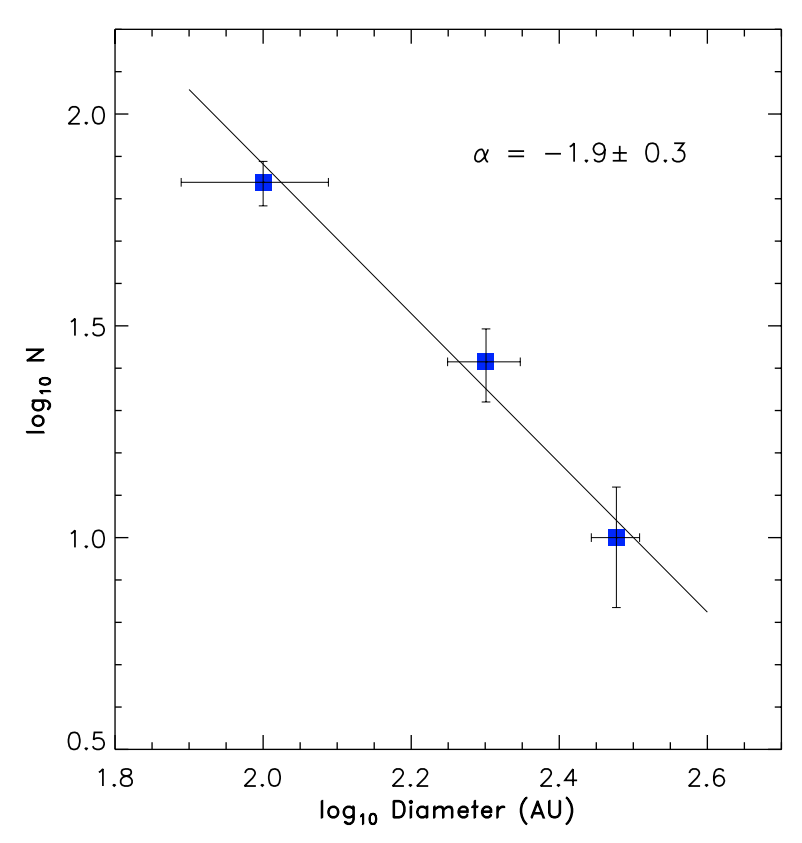

Fig. 6. Power-law fit to the size distribution of disks for diameters between 100-400 AU. The data can be described by a power-law with an exponent of $-1.9 \pm 0.3$.

\section{Discussion}

An important result of this work is that there seems to exist one single population of disks well characterized by a power-law distribution. Albeit the young age of the Trapezium, and given that disk destruction is well underway, it is perhaps too late to tell if the present day disk size distribution is primordial or if it is a consequence of the massive star formation environment. Nevertheless, the simple existence of a well defined disk size distribution hints at the existence of a primordial disk size distribution. Intuitively one would argue that a disk initial mass and size would be proportional to stellar mass (continuum surveys performed in the $\mathrm{mm} / \mathrm{submm}$ domain suggest a tendency for the disk mass to increase with the stellar mass, e.g., Natta et al. 2000) but the results expressed in Fig. 8, that there is not an obvious correlation between disk size and stellar mass, are intriguing and suggest a more complex picture. Most likely there is a random disk destruction process, or a combination of processes, that could be responsible for this lack of correlation.

The most important disks destruction processes are 1) viscous accretion; 2) close stellar encounters; and 3) stellar winds. Several environment related disk destruction mechanisms (2 and 3) have recently been proposed in the literature: Bate et al. (2003) performed sophisticated hydrodynamical simulations of cluster formation and find that most circumstellar disks are severely truncated by dynamical encounters. On the other hand, Richling \& Yorke (2000), considering photoevaporation, high initial disk masses, and fixed distances to $\theta^{1}$ Ori $\mathrm{C}$, obtain the present radius and masses observed in Trapezium's proplyds, for a timescale consistent with the age of $\theta^{1}$ Ori C ( 0.5 Myr). Also, Scally \& Clarke (2001) performed numerical $N$-body simulations of the Orion Nebula cluster and

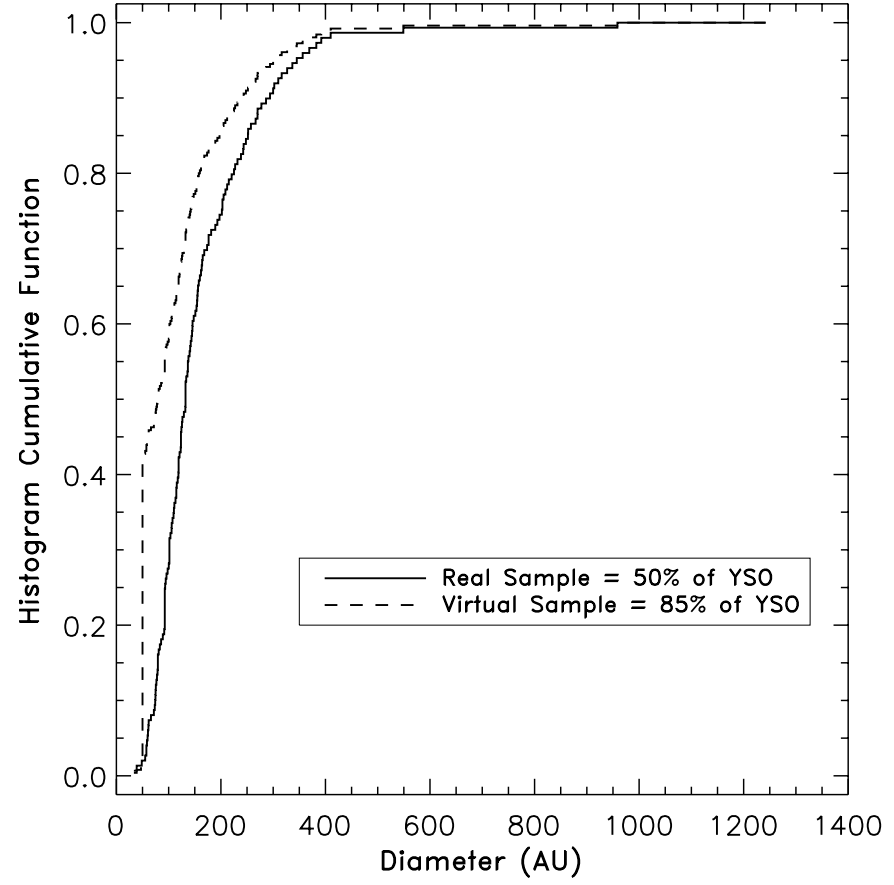

Fig. 7. Cumulative function of the histogram. The real sample is composed by the 149 disks in this paper and represents $\sim 50 \%$ of all Trapezium's YSO observed with the HST. The virtual sample is the corrected sample accounting for the 35\% of HST unresolved disks and is composed by our sample corrected by adding 106 disks with diameters less than $50 \mathrm{AU}$.

determine a very low probability of an encounter at a $d<$ $100 \mathrm{AU}$, at the cluster's present age, suggesting photoevaporation as the most significant disk destruction mechanism. If significant UV radiation is available, photoevaporation is the dominant disk dispersal mechanism (Hollenbach et al. 2000). The intense UV radiation field heats the disk surface, drives massloss and produces the bright ionization fronts, on a relatively short timescale. For example, the Trapezium proplyds have radial velocities of 24 to $30 \mathrm{~km} \mathrm{~s}^{-1}$ (Henney \& O'Dell 1999) and inferred disk masses of 0.005-0.02 $M_{\odot}$ (Lada et al. 1996; Bally et al. 1998b), with the $0.02 M_{\odot}$ upper bound determined for the largest pure silhouette 114-426. Mass-loss rates of order $10^{-7}$ to $10^{-6} M_{\odot} \mathrm{yr}^{-1}$ have been measured for a number of proplyds by several groups (Churchwell et al. 1987; Johnstone et al. 1997; Bally et al. 1998b; Johnstone et al. 1998; Henney \& Arthur 1998; Henney \& O’Dell 1999) with a mean mass-loss rate of $3.3 \times 10^{-7} M_{\odot} \mathrm{yr}^{-1}$ for all 31 proplyds that have been studied with sufficient detail (out of a total of $\sim 150$ Orion bright proplyds). This implies that a minimum Solar Nebula mass disk $\left(0.01 M_{\odot}\right)$ will loose half of its mass in $10^{4}$ to $10^{5}$ years and even less for the less massive observed objects. Such lifetimes are quite small compared to the estimated age of $\theta^{1}$ Ori $\mathrm{C}$ or 0.5 Myr. Herbig \& Terndrup (1986) and Hillenbrand (1997) find that the ages for low-mass stars in the Orion Nebula cluster range from $3 \times 10^{5}$ to $10^{6} \mathrm{yr}$, with only a few as young as $10^{5} \mathrm{yr}$. This brings a major conundrum, since proplyds exist and have been surviving the photoevaporation. There are two possible explanations: either $\theta^{1}$ Ori $\mathrm{C}$ is very young, or the illumination time of the proplyds is short (which would require a large 


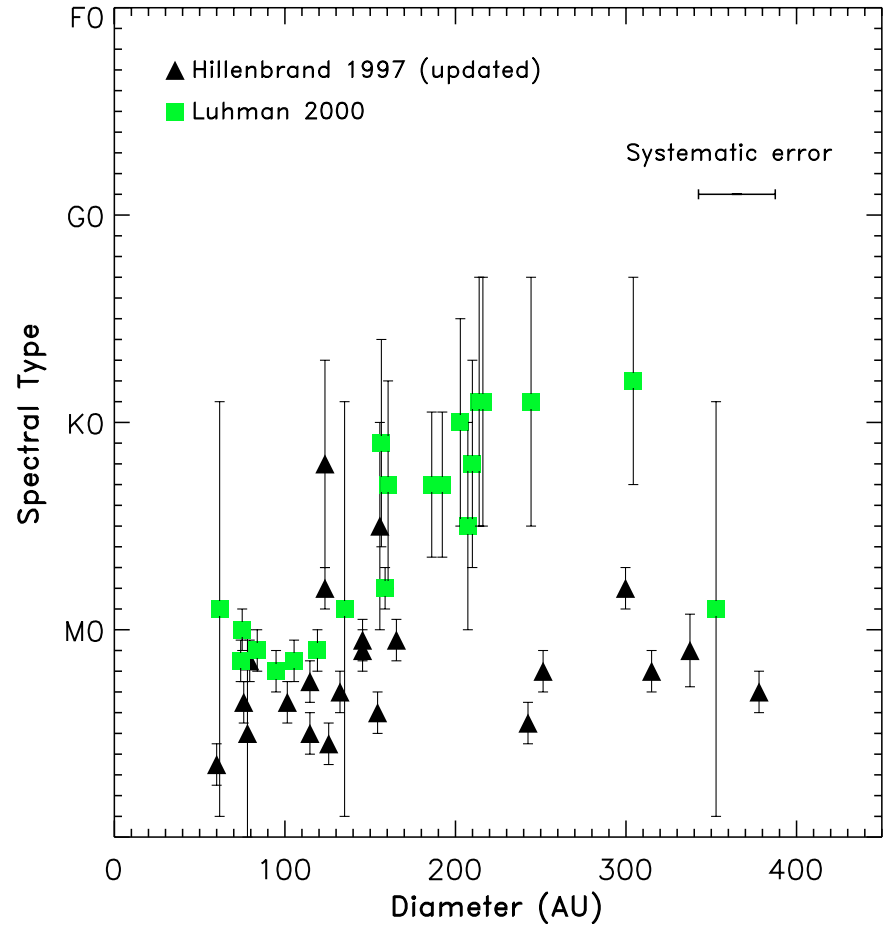

Fig. 8. Disk diameter versus spectral-type of central parent star. There is not an obvious correlation between the two variables.

spatial motion of $\theta^{1}$ Ori C). Recently, Tan (2004) claimed that the $\mathrm{BN}$ object is a runaway B star dynamical ejection event by the $\theta^{1}$ Ori $C$ star, just $\sim 4000$ years ago. Regardless, the point is that photoevaporation is likely to be the dominant disk destruction process. Still, relevant observational studies of the dynamics of embedded stellar clusters are not available yet (efficient near-infrared high-resolution spectrographs are only now becoming available) and it is too soon to dismiss close stellar encounters as an important disk destruction process in young clusters.

\subsection{The Trapezium as the birthplace of Solar system analogues}

\section{Can planet formation endure disk destruction mechanisms?}

If the growth of larger particles can occur before the removal of gas and small particles, planets may nevertheless form inside the dust disks embedded in such an adverse environment. There are evidences for grain growth in the largest silhouette 114-426 (Throop et al. 2001; Shuping et al. 2003). Recently, Throop \& Bally (2005) proposed that UV radiation can stimulate the rapid formation of planetesimals in externally-illuminated protoplanetary disks. It might then be that photoionization has an overall positive feedback on planet formation, while being an important (gas) disk destruction process. If this is the case, this would have far reaching implications to planet formation in the Galaxy, and likely in the Universe, since most stars are born in clusters containing $\mathrm{O}$ and B stars (Lada \& Lada 2003). In the specific case of the Trapezium, where about half of the disks are larger or about the Solar system size (see Sect. 3.7), one could expect planetary systems to be common and not fundamentally different from the Solar system itself.

It is striking that the remnant Solar system disk appears to be truncated, not unlike the Trapezium disk discussed in this paper. It is now a rather well established and intriguing fact that the Kuiper belt, which is a repository of the solar system's most primitive matter, has a well defined outer edge at about $50 \mathrm{AU}$ (e.g., Jewitt 2002). At least three mechanisms for its origin have been proposed, none of which has raised the general consensus of the community of the experts (see Morbidelli et al. 2003, for a review): 1) a dynamical origin involving a 1 Gyr lived Mars mass at object at $60 \mathrm{AU}$ (Brunini \& Melita 2002); 2) differential accretion rates producing a disk edge (Weidenschilling 2003); and 3) planetesimal disk truncation by the passage of a star in the vicinity of the Sun (e.g., Ida et al. 2000; Kobayashi \& Ida 2001). The HST images of the Trapezium cluster disks, in particular the silhouette disks, present us with clear evidence that these disks seem to have already well defined edges at their relatively very young ages. If the Sun was born in a stellar cluster, the probable case as most stars are born in clusters, this is suggestive that the origin of the Kuiper belt outer edge is likely to be due to the star formation environment and disk destruction processes (photoevaporation, collisions) present in archetypal star formation factories such as the Trapezium. If this is the case, these well defined outer edges are imprinted in these disks at the earliest phases of their evolution, even before the formation of Kuiper belt-like objects.

The results in this paper, together with future higherresolution observations and modeling, can help identify the dominant process for circumstellar disk destruction and provide insights into the survival rate of circumstellar material surrounding the YSOs, and therefore, insights on star and planet formation in very young clusters containing $\mathrm{O}$ and $\mathrm{B}$ stars, the typical nursery for most stars in the Galaxy.

\section{Conclusions}

The main results in this paper can be summarized as follow:

- There is no meaningful correlation between the IF "chord diameters" and the projected distance to $\theta^{1}$ Ori C. We find the same tendency in our analysis of previously published data.

- Direct measurements of 10 disks embedded in bright proplyds show a great dispersion in the diameter ratio $R=$ disk diameter/ IF diameter, and $R$ seems not to be correlated with the projected distance to $\theta^{1}$ Ori C. $244-440(R=15 \%)$ and $072-135(R=71 \%)$ illustrate the 2 opposite "extreme" cases.

- Assuming $R=49 \pm 16 \%$ (the average ratio from the 10 cases) to compute the disk sizes for the 125 bright proplyds we determined an unimodal disk size distribution, representing a single population of disks, that is well characterized by a power-law distribution with exponent of $-1.9 \pm 0.3$.

- For the stellar mass sampled (from late $G$ to late $M$ stars) we find that there is no obvious correlation between disk size and stellar mass. 
- The pure silhouettes are clearly the largest disks and have large projected distances to $\theta^{1}$ Ori $\mathrm{C}$. In particular, object $114-426$ is rather unique given its size and it falls off the above characterized disk size distribution.

- We estimate that about $40 \%$ to $45 \%$ of the Trapezium cluster disks have radius larger than $50 \mathrm{AU}$, the Solar System size.

- We suggest that the origin of the Solar system's (Kuiper belt) outer edge is likely to be due to the star formation environment and disk destruction processes (photoevaporation, collisions) present in the stellar cluster on which the Sun was probably formed.

- We identified a previously unknown proplyd and named it 266-557, following convention.

This statistical analysis should be repeated in function of "true" distances to the OB stars and not the projected ones. Proplyd geometry and true distances will lead to the $3 \mathrm{D}$ spatial distribution of proplyds in Orion.

Acknowledgements. The authors are grateful to Nicole Homeier, Michael Liu, Martino Romaniello, Herve Bouy and Ricardo Demarco for fruitful discussions.

\section{References}

Bally, J., Devine, D., \& Sutherland, R. 1995, Rev. Mex. Astron. Astrofis., 1, 19

Bally, J., Sutherland, R., Devine, D., et al. 1998a, AJ, 116, 293

Bally, J., Testi, L., Sargent, A., et al. 1998b, 116, 854

Bally, J., O'Dell, C. R., \& McCaughrean, M. J. 2000, AJ, 119, 2919

Bally, J., Licht, D., Smith, N., et al. 2005, AJ, 129, 355

Bate, M. R., Bonnell, I. A., \& Bromm, V. 2003, MNRAS, 339, 577

Brunini, A., \& Melita, M. 2002, Icarus 160, 32

Cohen, J. 1988, in Statistical power analysis for the behavioral sciences, 2nd ed., ed. Lawrence Erlbaum, New Jersey

Churchwell, E., et al. 1987, ApJ, 321, 516

Henney, W. J., \& Arthur, S. 1998, AJ, 116, 322

Henney, W. J., \& O’Dell, C. R. 1999, AJ, 118, 2350

Henney, W. J., Raga, A. C., Lizano, S., et al. 1996, ApJ, 465, 216

Herbig, G. H., \& Terndrup, D. M. 1986, 307, 609

Hillenbrand, L. A. 1997, AJ, 113, 1733

Hillenbrand, L. A., \& Carpenter, J. M. 2000, ApJ, 540, 236

Hillenbrand, L. A., \& Hartmann, L. W. 1998, ApJ, 492, 540

Hollenbach, D. J., Yorke, H. W., \& Johnstone, D. 2000, in Protostars and Planets IV, ed. V. Mannings, A. P. Boss, \& S. S. Russell (University of Arizona Press), 401

Ida, S., Larwood, J., \& Burkert, A. 2000, ApJ, 528, 351
Jewitt, D. 2002, in The Origins of Stars and Planets: The VLT View (Heidelberg: Springer Verlag), ed. J. Alves, \& M. McCaughrean, 405

Johnstone, D., Hollenbach, D., \& Bally, J. 1998, ApJ, 499, 758

Kobayashi, H., \& Ida, S. 2001, Icarus, 153, 416

Lada, C. J., \& Lada, E. 2003, ARA\&A, 41, 57

Lada, E. A., Dutrey, A., Guilloteau, S., et al. 1996, A\&AS, 189, L5301

Lada, C. J., Muench, A. A., Haisch, K. E., Jr., et al. 2000, AJ, 120, 3162

Luhman, K. L., Rieke, G. H., Young, E. T., et al. 2000, AJ, 540, 1016

McCaughrean, M. J., \& Stauffer, J. R. 1994, AJ, 108, 1382

McCaughrean, M. J., \& O’Dell, C. R. 1996, AJ, 111, 1977

McCaughrean, M. J., Chen, H., Bally, J., et al. 1998, ApJ, 429, L157

Morbidelli, A., Brown, M. E., \& Levison, H. F. 2004, Earth, Moon and Planets 92, 1

Muench, A. A., Lada, E. A., \& Lada, C. J. 2000, ApJ, 533, 358

Muench, A. A., Alves, J., Lada, C. J., et al. 2001, ApJ, 558, L51

Muench, A. A., Lada, E. A., Lada, C., et al. 2002, AJ, 573, 366

Natta, A., Grinin, V., \& Mannings, V. 2000, in Protostars and Planets IV, ed. V. Mannings, A. P. Boss, \& S. S. Russell (University of Arizona Press), 559

Yorke, H. W., \& Bodenheimer, P. 1999, ApJ, 525, 330

O’Dell, C. R. 1998, AJ, 115, 263

O’Dell, C. R. 2001a, PASP, 113, 29

O'Dell, C. R. 2001b, AJ, 122, 2662

O’Dell, C. R., \& Wen, Z. 1994, ApJ, 436, 194

O’Dell, C. R., \& Wong, K. 1996, AJ, 111, 846

O’Dell, C. R., Wen, Z., \& Hu, X. 1993, ApJ, 410, 696

Press, W. H., Teukolsky, S. A., Vetterling, W. T., \& Flannery, B. P. 1996, Numerical Recipes in Fortran 77 (Cambridge University Press), 630

Prosser, C. F., Stauffer, J. R., Hartmann, L., et al. 1994, AJ, 421, 517

Richling, S., \& Yorke, H. W. 2000, AJ, 539, 258

Rodmann J. 2002, Diploma Thesis, Univ. of Postdam, Germany

Scally, A., \& Clarke, C. 2001, MNRAS, 325, 449

Scally, A., Clarke, C., \& McCaughrean, M. J. 2005, MNRAS, 358, 742

Schertl, D., Balega, Y. Y., Preibisch, Th., et al. 2003, AA, 402, 267

Shuping, R. Y., Bally, J., Morris, M., et al. 2003, ApJ, 587, 109

Silverman, B.W. 1986, Density Estimation for Statistics and Data Analysis, ed. Chapman, \& Hall

Smith, N., Bally, J., Licht, D., et al. 2005, AJ, 129, 382

Störzer, H., \& Hollenbach, D. 1999, ApJ, 515, 669

Tan, J. C. 2004, ApJ, 607, L47

Throop, H. B., Bally, J., Esposito, L. W., et al. 2001, Science, 292, 1686

Throop, H. B., \& Bally, J. 2005, AJ, 623, 149

Weidenschilling, S. 2003, in Comet II, ed. Festou, et al. (Tucson: University of Arizona) 


\section{Online Material}


S. M. Vicente and J. Alves: Size distribution of circumstellar disks, Online Material p 2

Table 3. Catalog of 162 Orion proplyds observational properties.

\begin{tabular}{|c|c|c|c|c|c|c|c|c|}
\hline \multirow[t]{2}{*}{ Name } & \multirow{2}{*}{$\begin{array}{c}\mathrm{RA}^{a} \\
(\mathrm{~J} 2000.0)\end{array}$} & \multirow{2}{*}{$\begin{array}{c}\operatorname{Dec}^{a} \\
(\mathrm{~J} 2000.0)\end{array}$} & \multicolumn{2}{|c|}{ Disk diameter $^{b}$} & \multicolumn{2}{|c|}{ IF diameter ${ }^{b}$} & \multirow{2}{*}{$\begin{array}{r}d_{\text {proj }} \theta^{1} \text { OriC } \\
\left({ }^{\prime \prime}\right)\end{array}$} & \multirow[t]{2}{*}{ Comments $^{c}$} \\
\hline & & & (pix) & $(\mathrm{AU})$ & (pix) & $(\mathrm{AU})$ & & \\
\hline $005-514$ & 53500.72 & -52521.4 & 4.5 & 203 & 9.2 & 414 & 264.1 & \\
\hline $064-705$ & - & - & - & - & - & - & - & not found \\
\hline $066-652$ & 53506.82 & -52657.7 & 6.7 & 300 & 13.6 & 612 & 256.2 & \\
\hline $072-135$ & 53507.25 & -52136.9 & 7.5 & 336 & 10.5 & 472 & 176.3 & visible disk \\
\hline $073-227$ & 53507.35 & -52229.5 & 2.2 & 101 & 4.6 & 207 & 148.9 & \\
\hline $097-125$ & 53509.70 & $\begin{array}{lll}-521 & 26.5\end{array}$ & 2.2 & 101 & 4.6 & 207 & 155.8 & \\
\hline $102-021$ & 53510.16 & -52021.7 & 1.7 & 76 & 3.4 & 155 & 205.0 & \\
\hline $102-233$ & 53510.18 & -52234.8 & 2.6 & 118 & 5.4 & 241 & 107.4 & \\
\hline $106-156$ & 53510.60 & -52157.8 & 3.5 & 156 & 7.1 & 318 & 123.6 & \\
\hline $106-417$ & 53510.64 & -52419.6 & 2.0 & 90 & 4.1 & 184 & 103.8 & \\
\hline $109-247$ & 53510.95 & -52248.3 & 2.3 & 106 & 4.8 & 216 & 91.0 & \\
\hline $109-327$ & 53510.94 & -52326.6 & 3.1 & 141 & 6.4 & 288 & 83.7 & \\
\hline $109-449$ & 53510.60 & -52157.8 & 2.7 & 123 & 5.6 & 252 & 123.6 & \\
\hline $114-426$ & 53511.42 & -52429.2 & 27.6 & 1242 & - & - & 99.8 & silhouette \\
\hline $117-352$ & 53511.80 & -52354.0 & 5.1 & 229 & 10.4 & 468 & 76.4 & \\
\hline 119-340 & 53511.97 & -52342.0 & 1.3 & 59 & 2.7 & 121 & 70.2 & \\
\hline $121-1925$ & 53512.00 & -51924.3 & 8.0 & 360 & - & - & 247.4 & silhouette \\
\hline $121-434$ & 53512.21 & -52436.4 & 1.4 & 62 & 2.8 & 126 & 96.3 & \\
\hline $124-132$ & 53512.36 & -52132.1 & 6.0 & 270 & 12.3 & 552 & 127.3 & \\
\hline $127-711$ & 53512.80 & -52708.3 & 1.7 & 75 & 3.4 & 153 & 229.2 & \\
\hline $128-044$ & 53512.77 & -52043.6 & 3.4 & 155 & 7.0 & 317 & 168.7 & \\
\hline $131-046$ & 53513.02 & -52046.0 & 2.9 & 132 & 6.0 & 270 & 165.3 & \\
\hline $131-247$ & 53513.13 & -52248.5 & 2.1 & 93 & 4.2 & 189 & 61.6 & \\
\hline $132-221$ & 53513.17 & -52222.0 & 0.7 & 33 & 1.5 & 68 & 79.2 & \\
\hline $132-222$ & 53513.17 & -52222.2 & 0.9 & 44 & 2.0 & 90 & 79.0 & \\
\hline $132-1832$ & 53513.21 & -51832.3 & 9.4 & 423 & - & - & 293.5 & silhouette; not found - BOM \\
\hline $135-220$ & 53513.52 & -52220.4 & 2.6 & 119 & 5.4 & 243 & 77.3 & \\
\hline $135-227$ & 53513.36 & -52227.2 & 1.6 & 73 & 3.3 & 148 & 73.6 & \\
\hline $137-222$ & 53513.73 & $\begin{array}{ll}-52222.8\end{array}$ & 1.6 & 74 & 3.4 & 151 & 73.4 & \\
\hline 138-207 & 53513.78 & -52207.6 & 3.1 & 139 & 6.3 & 283 & 85.9 & \\
\hline $139-320$ & 53513.95 & -52321.5 & 1.3 & 57 & 2.6 & 117 & 38.2 & \\
\hline $140-1952$ & 53513.95 & -51951.1 & 2.5 & 112 & 5.1 & 228 & 214.4 & \\
\hline $141-301$ & 53514.18 & -52302.1 & 7.1 & 317 & 14.4 & 648 & 40.7 & \\
\hline $141-520$ & 53514.15 & -52522.8 & 5.0 & 225 & 7.2 & 326 & 123.1 & visible disk \\
\hline $143-425$ & 53514.33 & -52426.5 & 3.0 & 137 & 6.2 & 279 & 70.1 & \\
\hline $143-522$ & 53514.43 & -52524.5 & 5.8 & 263 & 14.4 & 648 & 123.7 & visible disk \\
\hline $144-334$ & 53514.42 & -52334.9 & 1.9 & 84 & 3.8 & 171 & 32.8 & \\
\hline $147-323$ & 53514.74 & $\begin{array}{ll}-5 & 2323.9\end{array}$ & 3.9 & 176 & 8.0 & 360 & 26.2 & \\
\hline $149-329$ & 53514.95 & -52330.1 & 1.4 & 62 & 2.8 & 126 & 23.8 & \\
\hline $150-231$ & 53515.01 & -52231.5 & 1.8 & 82 & 3.7 & 168 & 56.1 & \\
\hline $152-319$ & 53515.23 & $\begin{array}{lll}-5 & 23 & 19.6\end{array}$ & 2.2 & 101 & 4.6 & 207 & 18.9 & \\
\hline $152-738$ & 53515.36 & -52741.3 & 2.6 & 119 & 5.4 & 243 & 256.2 & \\
\hline $153-1902$ & - & - & - & - & - & - & - & not found \\
\hline $153-321$ & 53515.37 & -52322.2 & 1.3 & 57 & 2.6 & 117 & 16.8 & \\
\hline $154-225$ & 53515.35 & -52225.6 & 1.9 & 87 & 4.0 & 178 & 59.8 & \\
\hline $154-240$ & 53515.38 & -52240.5 & 3.9 & 176 & 8.0 & 360 & 45.7 & \\
\hline $154-324$ & 53515.38 & $\begin{array}{lll}-5 & 2325.0\end{array}$ & 1.6 & 71 & 3.2 & 144 & 16.4 & \\
\hline $155-338$ & 53515.55 & -52338.3 & 4.5 & 203 & 9.2 & 414 & 20.2 & \\
\hline $156-403$ & 53515.65 & -52404.2 & 2.1 & 93 & 4.2 & 189 & 42.2 & \\
\hline $157-323$ & 53515.73 & -52323.2 & 2.8 & 125 & 5.7 & 256 & 11.3 & \\
\hline $157-533$ & 53515.76 & -52535.2 & 2.7 & 123 & 5.6 & 252 & 131.1 & \\
\hline $158-323$ & 53515.84 & $\begin{array}{lll}-5 & 2323.1\end{array}$ & 4.7 & 210 & 9.5 & 428 & 9.5 & \\
\hline $158-326$ & 53515.86 & -52326.2 & 4.8 & 216 & 9.8 & 441 & 9.5 & \\
\hline $158-327$ & 53515.81 & $\begin{array}{ll}-5 & 2327.2\end{array}$ & 5.4 & 243 & 11.0 & 495 & 10.5 & \\
\hline $158-425$ & - & - & - & - & - & - & - & not found \\
\hline $159-338$ & 53515.92 & -52338.8 & 2.1 & 93 & 4.2 & 189 & 17.4 & \\
\hline $159-350$ & 53515.97 & -52350.9 & 6.8 & 304 & 13.8 & 621 & 28.3 & \\
\hline
\end{tabular}


S. M. Vicente and J. Alves: Size distribution of circumstellar disks, Online Material p 3

Table 3. continued.

\begin{tabular}{|c|c|c|c|c|c|c|c|c|}
\hline \multirow[t]{2}{*}{ Name } & \multirow{2}{*}{$\begin{array}{c}\mathrm{RA}^{a} \\
(\mathrm{~J} 2000.0)\end{array}$} & \multirow{2}{*}{$\begin{array}{c}\operatorname{Dec}^{a} \\
(\mathrm{~J} 2000.0)\end{array}$} & \multicolumn{2}{|c|}{ Disk diameter ${ }^{b}$} & \multicolumn{2}{|c|}{ IF Diameter $^{b}$} & \multirow{2}{*}{$\begin{array}{r}d_{\text {proj }} \theta^{1} \text { OriC } \\
\left({ }^{\prime \prime}\right)\end{array}$} & \multirow[t]{2}{*}{ Comments $^{c}$} \\
\hline & & & (pix) & $(\mathrm{AU})$ & (pix) & $(\mathrm{AU})$ & & \\
\hline $159-418$ & 53515.94 & -52418.9 & 2.6 & 119 & 5.4 & 243 & 55.6 & \\
\hline $160-353$ & 53516.02 & -52353.9 & 4.3 & 192 & 8.7 & 392 & 31.0 & \\
\hline $161-307$ & 53516.06 & -52307.5 & 3.2 & 146 & 6.6 & 298 & 16.9 & \\
\hline $161-314$ & 53516.11 & -52314.7 & 2.6 & 116 & 5.3 & 238 & 10.2 & \\
\hline $161-324$ & 53516.08 & -52324.9 & 3.9 & 176 & 8.0 & 360 & 6.0 & \\
\hline $161-328$ & 53516.08 & -52328.4 & 3.0 & 137 & 6.2 & 279 & 7.7 & \\
\hline $163-026$ & 53516.19 & -52024.2 & 5.0 & 225 & - & - & 178.0 & silhouette \\
\hline $163-222$ & 53516.28 & -52221.4 & 2.8 & 127 & 6.0 & 268 & 61.4 & visible disk \\
\hline $163-249$ & 53516.32 & -52249.2 & 2.3 & 105 & 4.8 & 215 & 33.9 & \\
\hline $163-317$ & 53516.27 & -52316.7 & 4.1 & 186 & 8.4 & 380 & 7.1 & \\
\hline $163-322$ & - & - & - & - & - & - & - & not found - star feature \\
\hline $163-323$ & 53516.32 & -52323.2 & 2.9 & 132 & 6.0 & 270 & 2.2 & \\
\hline $164-511$ & 53516.42 & -52511.2 & 1.8 & 79 & 3.6 & 162 & 107.0 & \\
\hline $165-235$ & 53516.45 & -52235.5 & 3.3 & 150 & 6.8 & 306 & 47.4 & \\
\hline $165-254$ & 53516.53 & -52253.8 & 3.2 & 144 & - & - & 29.2 & silhouette \\
\hline $166-250$ & 53516.57 & -52250.3 & 1.7 & 77 & 3.5 & 157 & 32.6 & \\
\hline $166-316$ & 53516.61 & -52316.3 & 2.2 & 101 & 4.6 & 207 & 7.1 & \\
\hline $166-519$ & - & - & - & - & - & - & - & not found \\
\hline $167-231$ & 53516.70 & -52231.0 & 5.6 & 252 & - & - & 51.8 & silhouette \\
\hline $167-317$ & 53516.74 & -52316.6 & 5.4 & 244 & 11.1 & 499 & 7.6 & \\
\hline $167-325$ & 53516.72 & -52325.5 & 3.7 & 165 & 7.5 & 337 & 4.6 & \\
\hline $168-235$ & 53516.79 & -52234.3 & 2.4 & 110 & 5.0 & 224 & 48.7 & \\
\hline $168-326 \mathrm{NW}$ & - & - & - & - & - & - & - & not included \\
\hline 168-326SE & - & - & - & - & - & - & - & not included \\
\hline 168-328 & 53516.76 & -52328.3 & 3.2 & 143 & 6.5 & 292 & 7.0 & \\
\hline $169-338$ & 53516.89 & -52338.5 & 2.4 & 110 & 5.0 & 225 & 16.6 & \\
\hline $169-549$ & 53517.02 & -52548.8 & 1.6 & 74 & 3.4 & 152 & 144.7 & \\
\hline $170-249$ & 53516.95 & -52248.6 & 4.6 & 207 & 9.4 & 423 & 35.0 & \\
\hline $170-337$ & 53516.99 & -52337.4 & 5.6 & 251 & 11.4 & 513 & 16.3 & \\
\hline $171-315$ & 53517.10 & -52315.5 & 3.6 & 163 & 7.4 & 333 & 12.1 & not found - OW94 \\
\hline $171-334$ & 53517.07 & -52334.4 & 3.5 & 156 & 7.1 & 319 & 14.5 & \\
\hline $171-340$ & 53517.07 & -52340.2 & 3.5 & 159 & 7.2 & 324 & 19.2 & \\
\hline $171-434$ & 53517.16 & -52435.3 & 1.8 & 79 & 3.6 & 162 & 72.4 & \\
\hline $172-028$ & 53517.22 & -52028.1 & 6.0 & 270 & - & - & 173.9 & silhouette; not found - BOM \\
\hline $172-327$ & 53517.20 & -52326.9 & 2.9 & 132 & 6.0 & 270 & 11.9 & \\
\hline $173-236$ & 53517.32 & -52235.6 & 4.7 & 214 & 9.7 & 436 & 48.8 & \\
\hline $173-341$ & 53517.34 & -52341.7 & 2.7 & 123 & 5.6 & 250 & 22.7 & \\
\hline $174-400$ & - & - & - & - & - & - & - & not found \\
\hline $174-414$ & 53517.40 & -52414.5 & 2.5 & 115 & 5.2 & 234 & 52.9 & \\
\hline $175-251$ & 53517.45 & -52251.1 & 2.3 & 97 & 4.4 & 198 & 34.9 & \\
\hline $175-355$ & 53517.55 & -52355.5 & 2.3 & 106 & 4.8 & 216 & 36.2 & \\
\hline $176-325$ & 53517.55 & -52324.9 & 3.6 & 160 & 7.3 & 328 & 16.5 & \\
\hline $176-543$ & 53517.61 & -52544.3 & 6.7 & 300 & 11.7 & 525 & 141.3 & visible disk \\
\hline $177-341$ & 53517.67 & -52341.1 & 7.8 & 353 & 16.0 & 720 & 25.5 & \\
\hline $177-444$ & 53517.76 & -52444.4 & 1.3 & 60 & 2.7 & 122 & 83.2 & \\
\hline $177-454$ & 53517.73 & -52454.9 & 2.8 & 126 & 5.7 & 256 & 93.2 & \\
\hline $177-541$ & 53517.78 & -52542.2 & 3.8 & 171 & 7.7 & 348 & 139.5 & \\
\hline $179-353$ & 53517.96 & -52353.7 & 2.1 & 93 & 4.2 & 189 & 38.0 & \\
\hline $179-536$ & - & - & - & - & - & - & - & not found \\
\hline $180-331$ & 53518.04 & -52330.7 & 4.5 & 201 & 9.1 & 410 & 25.2 & \\
\hline $181-247$ & 53518.05 & -52246.8 & 2.9 & 131 & 6.7 & 302 & 43.1 & visible disk \\
\hline $182-316$ & 53518.22 & -52315.3 & 2.1 & 95 & 4.3 & 193 & 27.6 & \\
\hline $182-332$ & 53518.18 & -52331.4 & 3.0 & 135 & - & - & 27.2 & silhouette \\
\hline $182-413$ & 53518.21 & -52413.4 & 6.4 & 288 & 15.1 & 678 & 56.7 & visible disk \\
\hline $183-405$ & 53518.33 & -52404.9 & 8.4 & 378 & - & - & 50.5 & silhouette \\
\hline $183-419$ & 53518.32 & -52419.5 & 3.6 & 163 & 7.4 & 333 & 62.8 & \\
\hline $183-439$ & 53518.31 & -52439.4 & 2.2 & 101 & 4.6 & 207 & 80.9 & \\
\hline
\end{tabular}


S. M. Vicente and J. Alves: Size distribution of circumstellar disks, Online Material p 4

Table 3. continued.

\begin{tabular}{|c|c|c|c|c|c|c|c|c|}
\hline \multirow[t]{2}{*}{ Name } & \multirow{2}{*}{$\begin{array}{c}\mathrm{RA}^{a} \\
(\mathrm{~J} 2000.0)\end{array}$} & \multirow{2}{*}{$\begin{array}{c}\operatorname{Dec}^{a} \\
(J 2000.0)\end{array}$} & \multicolumn{2}{|c|}{ Disk diameter $^{b}$} & \multicolumn{2}{|c|}{ IF Diameter ${ }^{b}$} & \multirow{2}{*}{$\begin{array}{r}d_{\text {proj }} \theta^{1} \text { OriC } \\
\left({ }^{\prime \prime}\right)\end{array}$} & \multirow[t]{2}{*}{ Comments $^{c}$} \\
\hline & & & (pix) & $(\mathrm{AU})$ & (pix) & $(\mathrm{AU})$ & & \\
\hline $184-427$ & 53518.37 & -52427.2 & 2.5 & 115 & 5.2 & 234 & 70.0 & \\
\hline $184-520$ & 53518.49 & -52520.1 & 2.4 & 109 & 4.9 & 222 & 120.3 & \\
\hline $187-314$ & 53518.63 & -52313.4 & 2.7 & 123 & 5.6 & 252 & 34.0 & \\
\hline $189-329$ & 53518.85 & -52328.5 & 1.7 & 75 & 3.4 & 153 & 36.4 & \\
\hline $191-232$ & 53519.32 & -52235.6 & 2.2 & 99 & - & - & 63.6 & silhouette \\
\hline $191-350$ & 53519.05 & -52349.5 & 4.5 & 203 & 9.2 & 414 & 47.5 & \\
\hline $197-427$ & 53519.65 & -52426.7 & 7.5 & 337 & 12.1 & 543 & 79.9 & visible disk \\
\hline $198-222$ & 53519.75 & -52220.3 & 2.0 & 93 & 4.2 & 189 & 79.2 & \\
\hline $198-448$ & 53519.84 & -52448.2 & 3.2 & 145 & 6.6 & 297 & 99.3 & \\
\hline $201-534$ & 53520.18 & -52534.6 & 1.7 & 78 & 3.5 & 159 & 142.7 & \\
\hline $202-228$ & 53520.08 & -52227.0 & 2.5 & 115 & 5.2 & 234 & 77.7 & \\
\hline $203-504$ & 53520.27 & -52504.4 & 4.3 & 194 & 8.8 & 396 & 116.6 & \\
\hline $203-506$ & 53520.32 & -52505.9 & 6.0 & 270 & - & - & 118.3 & silhouette \\
\hline $205-052$ & 53520.37 & -52049.4 & 1.3 & 61 & 2.8 & 124 & 162.8 & \\
\hline $205-330$ & 53520.42 & -52328.8 & 3.0 & 137 & 6.2 & 279 & 60.3 & brighter object \\
\hline $205-421$ & 53520.52 & -52421.0 & 2.7 & 123 & 5.6 & 252 & 84.6 & not found - BOM \\
\hline $206-446$ & 53520.62 & -52446.5 & 7.0 & 315 & 16.3 & 734 & 104.6 & visible disk \\
\hline $208-122$ & - & - & - & - & - & - & - & not found \\
\hline $212-260$ & 53521.18 & -52258.1 & 2.1 & 94 & 4.3 & 192 & 75.2 & \\
\hline $212-557$ & 53521.18 & -52557.6 & 3.2 & 145 & 6.6 & 297 & 170.0 & \\
\hline $213-346$ & 53521.26 & -52345.1 & 1.3 & 57 & 2.6 & 117 & 76.2 & \\
\hline $215-317$ & - & - & - & - & - & - & - & not found \\
\hline $218-339$ & 53521.72 & -52338.0 & 2.0 & 93 & 4.2 & 189 & 81.2 & \\
\hline $218-354$ & 53521.76 & -52352.7 & 15.0 & 675 & - & - & 85.9 & silhouette \\
\hline $218-529$ & 53521.83 & -52528.5 & 5.6 & 252 & - & - & 149.6 & silhouette \\
\hline $221-433$ & - & - & - & - & - & - & - & not found \\
\hline $222-637$ & 53522.23 & -52638.1 & 3.7 & 165 & 7.5 & 337 & 213.2 & \\
\hline $224-728$ & 53522.43 & -52729.2 & 5.4 & 243 & 11.0 & 495 & 261.5 & \\
\hline $228-548$ & 53522.83 & -52547.6 & 2.0 & 93 & 4.2 & 189 & 173.8 & \\
\hline $231-460$ & 53523.01 & -52459.7 & 2.0 & 93 & 4.2 & 189 & 138.9 & \\
\hline $231-502$ & 53523.12 & -52501.7 & 2.0 & 93 & 4.2 & 189 & 141.3 & \\
\hline $232-453$ & 53523.19 & -52452.2 & 1.8 & 79 & 3.6 & 162 & 135.7 & \\
\hline $236-527$ & 53523.57 & -52526.0 & 2.9 & 132 & 6.0 & 270 & 163.7 & \\
\hline $237-627$ & 53523.65 & -52627.2 & 2.9 & 132 & 6.0 & 270 & 214.0 & \\
\hline 239-334 & 53523.78 & -52331.9 & 5.2 & 234 & - & - & 111.4 & silhouette \\
\hline $239-510$ & 53523.94 & -52509.2 & 3.1 & 141 & 6.4 & 288 & 155.5 & \\
\hline $240-314$ & 53523.93 & -52311.5 & 1.2 & 53 & 2.4 & 108 & 113.5 & \\
\hline $242-519$ & 53524.21 & -52517.9 & 3.4 & 152 & 6.9 & 310 & 164.8 & \\
\hline $244-440$ & 53524.38 & -52438.5 & 8.6 & 387 & 56.0 & 2520 & 142.3 & visible disk; giant proplyd \\
\hline $245-502$ & 53524.46 & -52500.5 & 3.4 & 154 & 7.0 & 315 & 156.0 & \\
\hline $245-632$ & 53524.45 & -52631.4 & 3.4 & 154 & 7.0 & 315 & 224.0 & \\
\hline $247-436$ & 53524.63 & -52434.3 & 2.9 & 132 & 6.0 & 270 & 143.3 & \\
\hline $250-439$ & 53524.96 & -52437.0 & 2.2 & 101 & 4.6 & 207 & 149.0 & \\
\hline $252-457$ & 53525.15 & -52456.0 & 6.3 & 284 & 12.9 & 580 & 161.8 & \\
\hline $264-532$ & 53526.36 & -52530.2 & 1.8 & 79 & 3.6 & 162 & 197.2 & \\
\hline $266-557$ & 53526.58 & -52556.6 & 2.6 & 119 & 5.4 & 243 & 217.5 & \\
\hline $282-458$ & 53528.10 & -52455.8 & 8.8 & 397 & 18.0 & 810 & 200.1 & \\
\hline $294-606$ & - & - & - & - & - & - & - & silhouette; out of image \\
\hline
\end{tabular}

${ }^{a}$ Units of right ascension are hours, minutes, seconds and units in declination are degrees, arcminutes and arcseconds (J2000.0).

$b$ The disk and IF diameters were determined directly from the data and do not account for finite size of the HST PSF.

$c$ Includes the reference for the 10 bright proplyds with visible embedded disks, the 16 pure silhouettes and the 14 objects listed in previous papers but not found in the $\mathrm{H}_{\alpha}$ image. For 4 of these objects, the IF and disk diameters were measured from their images published in the papers O’Dell \& Wen 1994 (OW94) and Bally et al. 2000 (BOM). 